\title{
Principal Component Analysis
}

\author{
Hervé Abdi • Lynne J. Williams
}

\begin{abstract}
Principal component analysis (PCA) is a multivariate technique that analyzes a data table in which observations are described by several inter-correlated quantitative dependent variables. Its goal is to extract the important information from the table, to represent it as a set of new orthogonal variables called principal components, and to display the pattern of similarity of the observations and of the variables as points in maps. The quality of the PCA model can be evaluated using cross-validation techniques such as the bootstrap and the jackknife. PCA can be generalized as correspondence analysis ( $\mathrm{CA}$ ) in order to handle qualitative variables and as multiple factor analysis (MFA) in order to handle heterogenous sets of variables. Mathematically, PCA depends upon the eigen-decomposition of positive semi-definite matrices and upon the singular value decomposition (SVD) of rectangular matrices.
\end{abstract}

\section{Keywords:}

Bilinear decomposition, correspondence analysis, cross-validation, eigen-decomposition, factor scores, jackknife, loadings, multicolinearity, multiple factor analysis, principal component analysis, predicted residual sum of squares, PRESS, residual sum of squares, RESS, singular value decomposition, small $N$ large $P$ problem.

Hervé Abdi

The University of Texas at Dallas

Lynne J. Williams

The University of Toronto Scarborough

Send correspondence to Hervé Abdi: herve@utdallas.edu www.utdallas.edu/ herve

We would like to thank Yoshio Takane for his very helpful comments on a draft of this paper. 


\section{Introduction}

Principal component analysis (PCA) is probably the most popular multivariate statistical technique and it is used by almost all scientific disciplines. It is also likely to be the oldest multivariate technique. In fact, its origin can be traced back to Pearson (1901) or even Cauchy (1829, see Grattan-Guinness, 1997, p. 416), or Jordan (1874, and also Cayley, Silverster, and Hamilton, see Stewart, 1993; Boyer and Merzbach, 1989, for more) but its modern instantiation was formalized by Hotelling (1933) who also coined the term principal component. PCA analyzes a data table representing observations described by several dependent variables, which are, in general, inter-correlated. Its goal is to extract the important information from the data table and to express this information as a set of new orthogonal variables called principal components. $\mathrm{PCA}_{\mathrm{CA}}$ also represents the pattern of similarity of the observations and the variables by displaying them as points in maps (see, for more details Jolliffe, 2002; Jackson, 1991; Saporta and Niang, 2009).

\section{Prerequisite notions and notations}

Matrices are denoted in upper case bold, vectors are denoted in lower case bold, and elements are denoted in lower case italic. Matrices, vectors, and elements from the same matrix all use the same letter $(e . g ., \mathbf{A}, \mathbf{a}, a)$. The transpose operation is denoted by the superscript ${ }^{\top}$. The identity matrix is denoted $\mathbf{I}$.

The data table to be analyzed by PCA comprises $I$ observations described by $J$ variables and it is represented by the $I \times J$ matrix $\mathbf{X}$, whose generic element is $x_{i, j}$. The matrix $\mathbf{X}$ has $\operatorname{rank} L$ where $L \leq \min \{I, J\}$.

In general, the data table will be pre-processed before the analysis. Almost always, the columns of $\mathbf{X}$ will be centered so that the mean of each column is equal to 0 (i.e., $\mathbf{X}^{\top} \mathbf{1}=\mathbf{0}$, where $\mathbf{0}$ is a $J$ by 1 vector of zeros and $\mathbf{1}$ is an $I$ by 1 vector of ones). If in addition, each element of $\mathbf{X}$ is divided by $\sqrt{I}$ ( or $\sqrt{I-1}$ ), the analysis is referred to as a covariance PCA because, in this case, the matrix $\mathbf{X}^{\top} \mathbf{X}$ is a covariance matrix. In addition to centering, when the variables are measured with different units, it is customary to standardize each variable to unit norm. This is obtained by dividing each variable by its norm (i.e., the square root of the sum of all the squared elements of this variable). In this case, the analysis is referred to as a correlation PCA because, then, the matrix $\mathbf{X}^{\top} \mathbf{X}$ is a correlation matrix (most statistical packages use correlation preprocessing as a default). 
The matrix $\mathbf{X}$ has the following singular value decomposition (SVD, see Abdi, 2007a,b; Takane, 2002, and Appendix B for an introduction to the SVD):

$$
\mathbf{X}=\mathbf{P} \Delta \mathbf{Q}^{\top}
$$

where $\mathbf{P}$ is the $I \times L$ matrix of left singular vectors, $\mathbf{Q}$ is the $J \times L$ matrix of right singular vectors, and $\boldsymbol{\Delta}$ is the diagonal matrix of singular values. Note that $\boldsymbol{\Delta}^{2}$ is equal to $\boldsymbol{\Lambda}$ which is the diagonal matrix of the (non-zero) eigenvalues of $\mathbf{X}^{\top} \mathbf{X}$ and $\mathbf{X X}^{\top}$.

The inertia of a column is defined as the sum of the squared elements of this column and is computed as

$$
\gamma_{j}^{2}=\sum_{i}^{I} x_{i, j}^{2} .
$$

The sum of all the $\gamma_{j}^{2}$ is denoted $\mathcal{I}$ and it is called the inertia of the data table or the total inertia. Note that the total inertia is also equal to the sum of the squared singular values of the data table (see Appendix A).

The center of gravity of the rows (also called centroid or barycenter, see Abdi, 2009 ), denoted $\mathbf{g}$, is the vector of the means of each column of $\mathbf{X}$. When $\mathbf{X}$ is centered, its center of gravity is equal to the $1 \times J$ row vector $\mathbf{0}^{\top}$.

The (Euclidean) distance of the $i$-th observation to $\mathbf{g}$ is equal to

$$
d_{i, \mathbf{g}}^{2}=\sum_{j}^{J}\left(x_{i, j}-g_{j}\right)^{2} .
$$

When the data are centered, Equation 3 reduces to

$$
d_{i, \mathbf{g}}^{2}=\sum_{j}^{J} x_{i, j}^{2} .
$$

Note that the sum of all $d_{i, \mathrm{~g}}^{2}$ is equal to $\mathcal{I}$ which is the inertia of the data table

\section{Goals of PCA}

The goals of PCA are to (a) extract the most important information from the data table, (b) compress the size of the data set by keeping only this important information, (c) simplify the description of the data set, and (d) analyze the structure of the observations and the variables. 
In order to achieve these goals, PCA computes new variables called principal components which are obtained as linear combinations of the original variables. The first principal component is required to have the largest possible variance (i.e., inertia and therefore this component will "explain" or "extract" the largest part of the inertia of the data table). The second component is computed under the constraint of being orthogonal to the first component and to have the largest possible inertia. The other components are computed likewise (see Appendix A.3 for proof). The values of these new variables for the observations are called factor scores, these factors scores can be interpreted geometrically as the projections of the observations onto the principal components.

\subsection{Finding the components}

In $\mathrm{PCA}$, the components are obtained from the singular value decomposition of the data table $\mathbf{X}$. Specifically, with $\mathbf{X}=\mathbf{P} \boldsymbol{\Delta} \mathbf{Q}^{\top}$ ( $c f$. Equation 1 ), the $I \times L$ matrix of factor scores, denoted $\mathbf{F}$ is obtained as

$$
\mathbf{F}=\mathbf{P} \boldsymbol{\Delta}
$$

The matrix $\mathbf{Q}$ gives the coefficients of the linear combinations used to compute the factors scores. This matrix can also be interpreted as a projection matrix because multiplying $\mathbf{X}$ by $\mathbf{Q}$ gives the values of the projections of the observations on the principal components. This can be shown by combining Equations 1 and 5 as:

$$
\mathbf{F}=\mathbf{P} \Delta=\mathbf{P} \Delta \mathrm{QQ}^{\top}=\mathrm{XQ}
$$

The components can also be represented geometrically by the rotation of the original axes. For example, if $\mathbf{X}$ represents 2 variables, the length of a word $(Y)$ and the number of lines of its dictionary definition $(W)$, such as the data shown in Table 1, then PCA represents these data by two orthogonal factors. The geometric representation of PCA is shown in Figure 1. In this figure, we see that the factor scores give the length (i.e., distance to the origin) of the projections of the observations on the components. This procedure is further illustrated in Figure 2. In this context, the matrix $\mathbf{Q}$ is interpreted as a matrix of direction cosines (because $\mathbf{Q}$ is orthonormal). The matrix $\mathbf{Q}$ is also called a loading matrix. In this context, the matrix $\mathbf{X}$ can be interpreted as the product of the factors score matrix by the loading matrix as:

$$
\mathbf{X}=\mathbf{F Q}^{\top} \quad \text { with } \mathbf{F}^{\top} \mathbf{F}=\boldsymbol{\Delta}^{2} \text { and } \mathbf{Q}^{\top} \mathbf{Q}=\mathbf{I}
$$

This decomposition is often called the bilinear decomposition of $\mathbf{X}$ (see, e.g., Kruskal, 1978). 


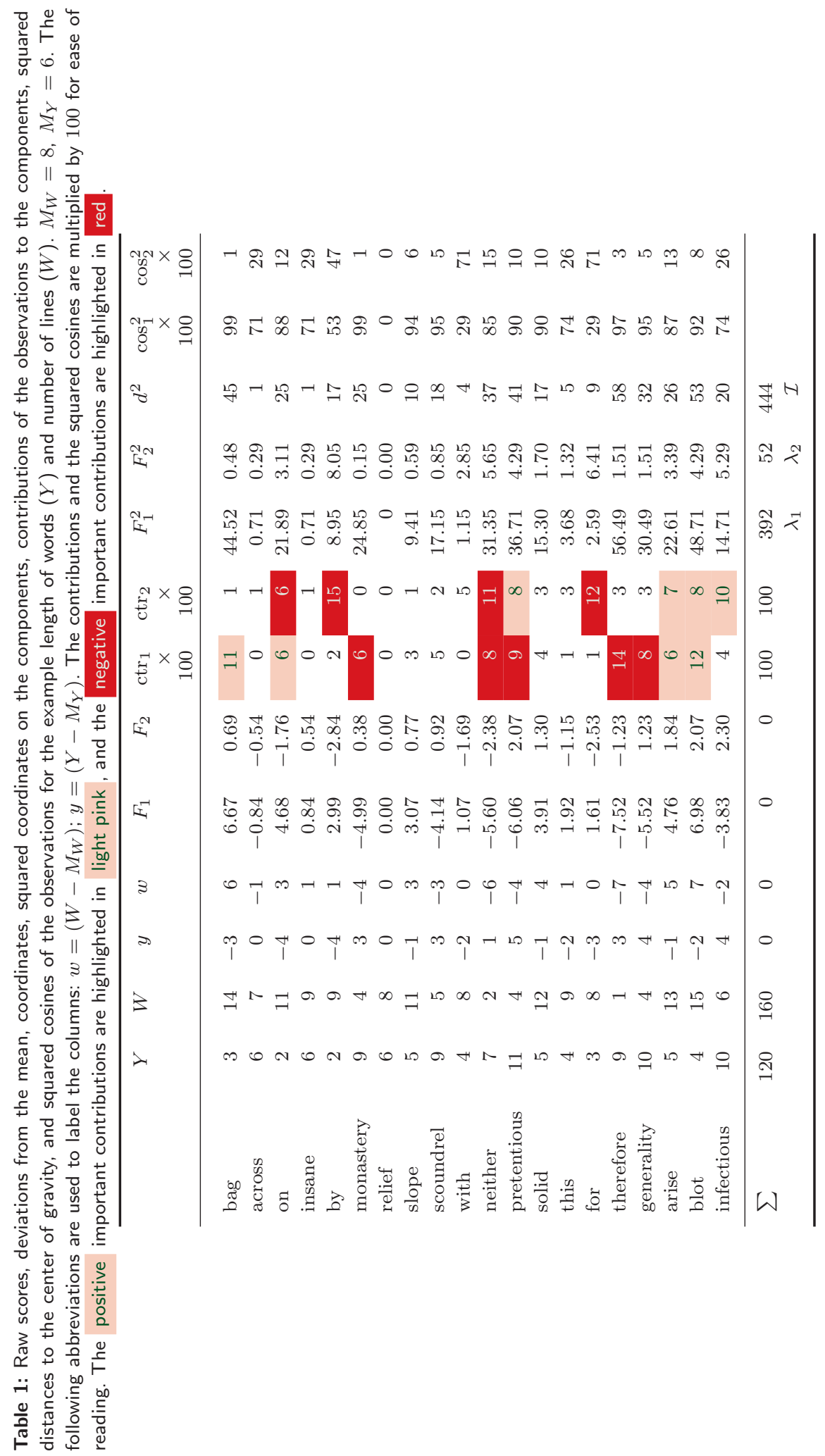




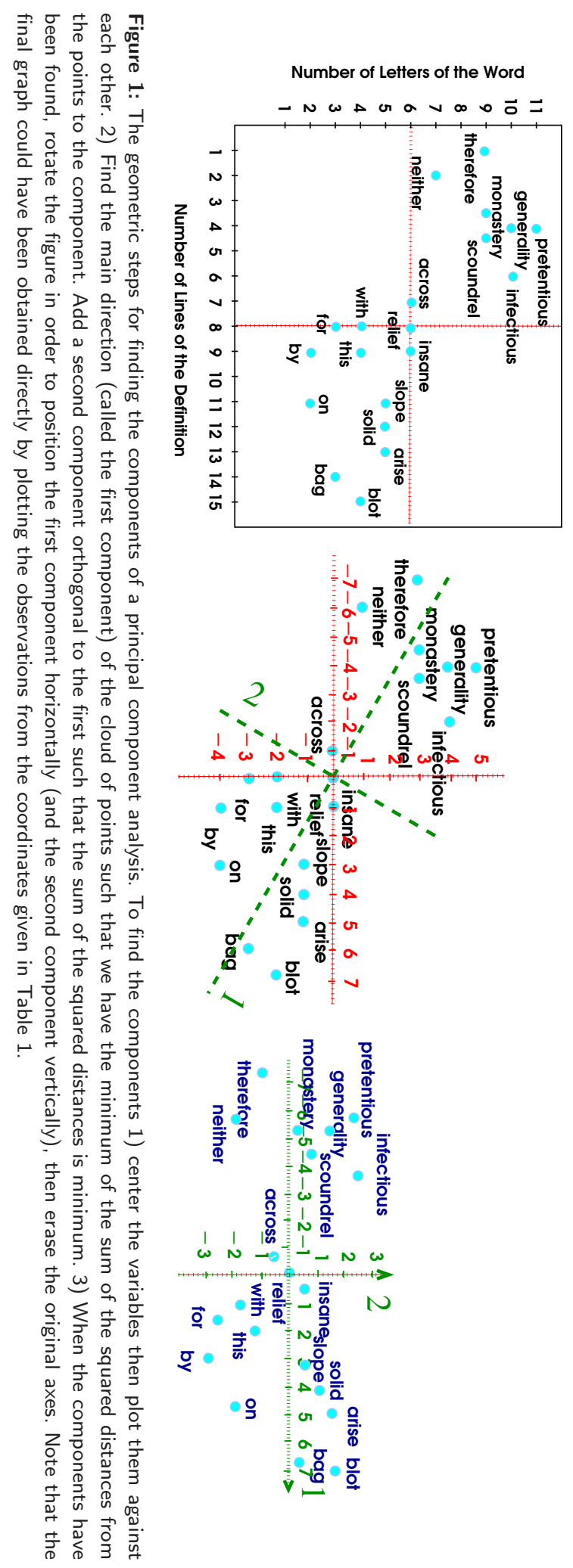




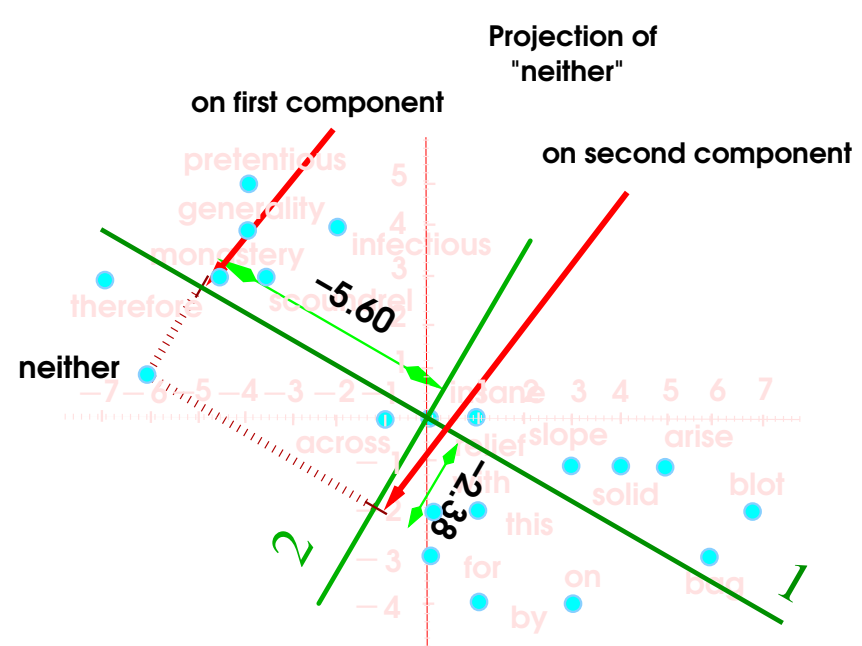

Figure 2: Plot of the centered data, with the first and second components. The projections (or coordinates) of the word "neither" on the first and the second components are equal to -5.60 and -2.38 .

\subsubsection{Projecting new observations onto the components}

Equation 6 shows that matrix $\mathbf{Q}$ is a projection matrix which transforms the original data matrix into factor scores. This matrix can also be used to compute factor scores for observations that were not included in the PCA. These observations are called supplementary or illustrative observations. By contrast, the observations actually used to compute the PCA are called active observations. The factor scores for supplementary observations are obtained by first positioning these observations into the PCA space and then projecting them onto the principal components. Specifically a $1 \times J$ row vector $\mathbf{x}_{\text {sup }}^{\top}$, can be projected into the PCA space using Equation 6 . This gives the $1 \times L$ vector of factor scores denoted $\mathbf{f}_{\text {sup }}^{\top}$ which is computed as:

$$
\mathbf{f}_{\text {sup }}^{\top}=\mathbf{x}_{\text {sup }}^{\top} \mathbf{Q} \text {. }
$$

If the data table has been preprocessed (e.g., centered or normalized) the same pre-processing should be applied to the supplementary observations prior to the computation of their factor scores.

As an illustration, suppose that - in addition to the data presented in Table $1-$ we have the French word "sur" (it means "on"). It has $Y_{\text {sur }}=3$ letters, and our French dictionary reports that its definition has $W_{\text {sur }}=12$ lines. Because sur is not an English word, we do not want to include it in the analysis, but we would like to know how it relates to the English vocabulary. So, we decided to treat this word as a supplementary observation. 


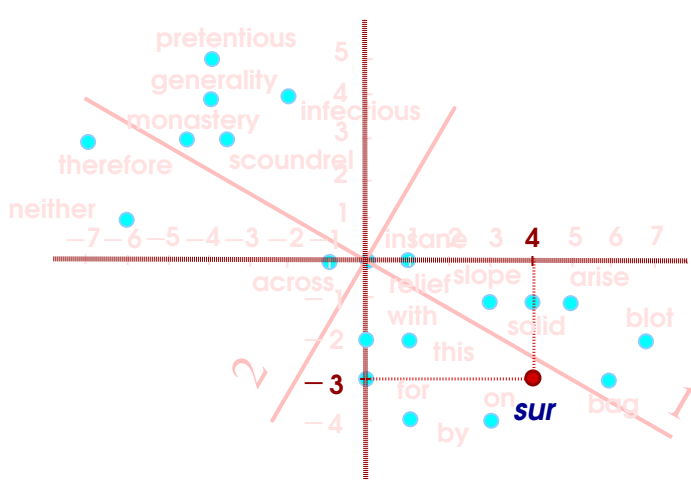

(a)

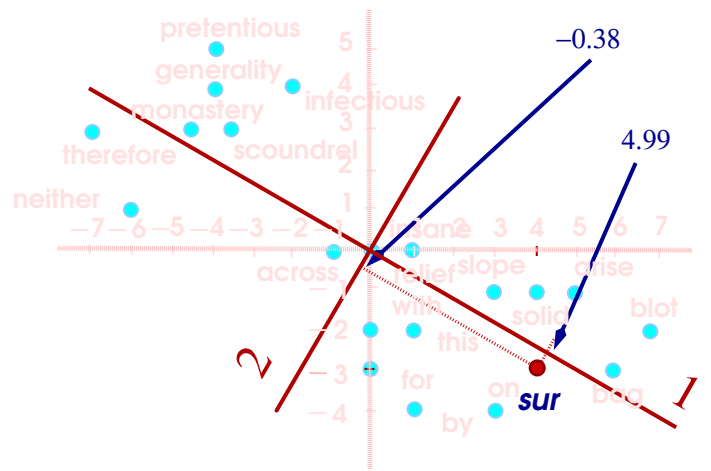

(b)

Figure 3: How to find the coordinates (i.e., factor scores) on the principal components of a supplementary observation: (a) the French word sur is plotted in the space of the active observations from its deviations to the $W$ and $Y$ variables; and (b) The projections of the sur on the principal components give its coordinates.

The first step is to preprocess this supplementary observation in a identical manner to the active observations. Because the data matrix was centered, the values of this observation are transformed into deviations from the English center of gravity. We find the following values:

$$
y_{\mathrm{sur}}=Y_{\mathrm{sur}}-M_{Y}=3-6=-3 \quad \text { and } \quad w_{\mathrm{sur}}=W_{\mathrm{sur}}-M_{W}=12-8=4 .
$$

Then we plot the supplementary word in the graph that we have already used for the active analysis. Because the principal components and the original variables are in the same space, the projections of the supplementary observation give its coordinates (i.e., factor scores) on the components. This is shown in Figure 3. Equivalently, the coordinates of the projections on the components can be directly computed from Equation 8 (see also Table 3 for the values of $\mathbf{Q}$ ) as:

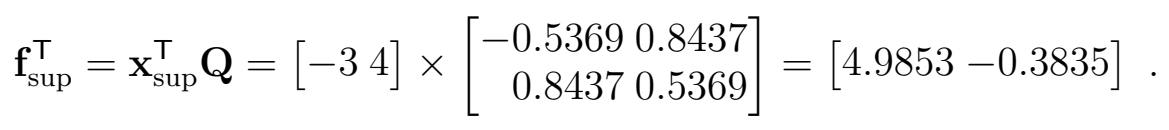

\section{Interpreting PCA}

\subsection{Contribution of an observation to a component}

Recall that the eigenvalue associated to a component is equal to the sum of the squared factor scores for this component. Therefore, the importance of an observation for a component can be obtained by the ratio of the squared factor score 
of this observation by the eigenvalue associated with that component. This ratio is called the contribution of the observation to the component. Formally, the contribution of observation $i$ to component $\ell$ is denoted $\operatorname{ctr}_{i, \ell}$, it is obtained as

$$
\operatorname{ctr}_{i, \ell}=\frac{f_{i, \ell}^{2}}{\sum_{i} f_{i, \ell}^{2}}=\frac{f_{i, \ell}^{2}}{\lambda_{\ell}}
$$

where $\lambda_{\ell}$ is the eigenvalue of the $\ell$ th component. The value of a contribution is between 0 and 1 and, for a given component, the sum of the contributions of all observations is equal to 1 . The larger the value of the contribution, the more the observation contributes to the component. A useful heuristic is to base the interpretation of a component on the observations whose contribution is larger than the average contribution (i.e., observations whose contribution is larger than $1 / I)$. The observations with high contributions and different signs can then be opposed to help interpret the component because these observations represent the two endpoints of this component.

The factor scores of the supplementary observations are not used to compute the eigenvalues and therefore their contributions are generally not computed.

\subsection{Squared Cosine of a component with an observation}

The squared cosine shows the importance of a component for a given observation. The squared cosine indicates the contribution of a component to the squared distance of the observation to the origin. It corresponds to the square of the cosine of the angle from the right triangle made with the origin, the observation, and its projection on the component and is computed as

$$
\cos _{i, \ell}^{2}=\frac{f_{i, \ell}^{2}}{\sum_{\ell} f_{i, \ell}^{2}}=\frac{f_{i, \ell}^{2}}{d_{i, \mathbf{g}}^{2}}
$$

where $d_{i, \mathbf{g}}^{2}$ is the squared distance of a given observation to the origin. The squared distance, $d_{i, \mathrm{~g}}^{2}$, is computed (thanks to the Pythagorean theorem) as the sum of the squared values of all the factor scores of this observation ( $c f$. Equation 4). Components with a large value of $\cos _{i, \ell}^{2}$ contribute a relatively large portion to the total distance and therefore these components are important for that observation.

The distance to the center of gravity is defined for supplementary observations and the squared cosine can be computed and is meaningful. Therefore, the value of $\cos ^{2}$ can help find the components that are important to interpret both active and supplementary observations. 
Table 2: Eigenvalues and percentage of explained inertia by each component.

\begin{tabular}{lrrrr}
\hline Component & \multicolumn{1}{c}{$\begin{array}{l}\lambda_{i} \\
\text { (eigenvalue) }\end{array}$} & $\begin{array}{c}\text { Cumulated } \\
\text { (eigenvalues) }\end{array}$ & $\begin{array}{c}\text { Percent of } \\
\text { of inertia }\end{array}$ & $\begin{array}{c}\text { Cumulated } \\
\text { (percentage) }\end{array}$ \\
\hline 1 & 392 & 392 & 88.29 & 88.29 \\
2 & 52 & 444 & 11.71 & 100.00 \\
\hline
\end{tabular}

Table 3: Loadings (i.e., coefficients of correlation between variables and components) and squared loadings. The elements of matrix $\mathbf{Q}$ are also provided.

\begin{tabular}{|c|c|c|c|c|c|c|}
\hline \multirow[b]{2}{*}{ Component } & \multicolumn{2}{|c|}{ Loadings } & \multicolumn{2}{|c|}{ Squared Loadings } & \multicolumn{2}{|c|}{ Q } \\
\hline & $Y$ & $W$ & $Y$ & $W$ & $Y$ & $W$ \\
\hline 1 & -.9927 & -.9810 & .9855 & .9624 & -.5369 & .8437 \\
\hline 2 & .1203 & -.1939 & .0145 & .0376 & .8437 & .5369 \\
\hline$\sum$ & & & 1.0000 & 1.0000 & & \\
\hline
\end{tabular}

\subsection{Loading: correlation of a component and a variable}

The correlation between a component and a variable estimates the information they share. In the PCA framework, this correlation is called a loading. Note that the sum of the squared coefficients of correlation between a variable and all the components is equal to 1 . As a consequence, the squared loadings are easier to interpret than the loadings (because the squared loadings give the proportion of the variance of the variables explained by the components). Table 3 gives the loadings as well as the squared loadings for the word length and definition example.

It is worth noting that the term "loading" has several interpretations. For example, as previously mentioned, the elements of matrix $\mathbf{Q}$ ( $c f$. equation 58) are also called loadings. This polysemy is a potential source of confusion, and therefore it is worth checking what specific meaning of the word "loadings" has been chosen when looking at the outputs of a program or when reading papers on PCA. In general, however, the different meanings of "loadings" lead to equivalent interpretations of the components. This happens because the different types of loadings differ mostly by their type of normalization. For example, the correlations of the variables with the components are normalized such that the sum of the squared correlations of a given variable is equal to one; By contrast, the elements of $\mathbf{Q}$ are normalized such that the sum of the squared elements of a given component is equal to one. 


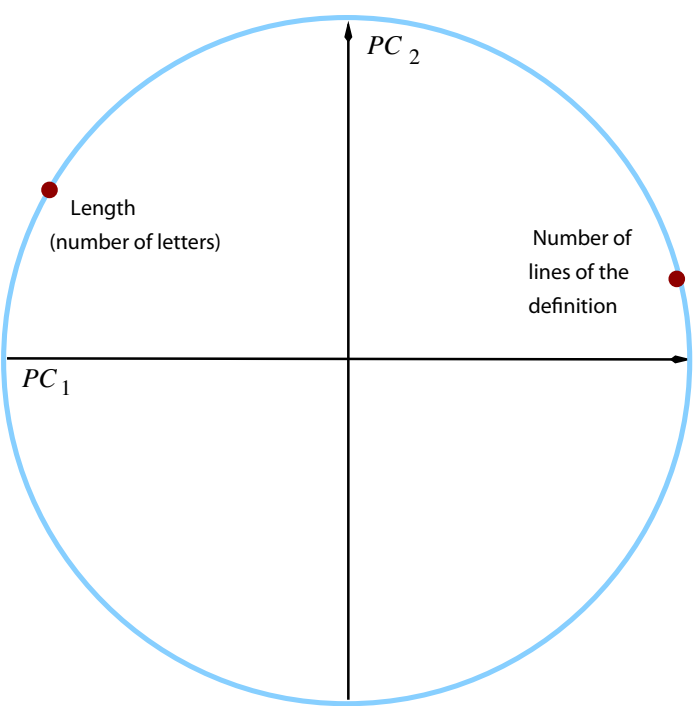

(a)

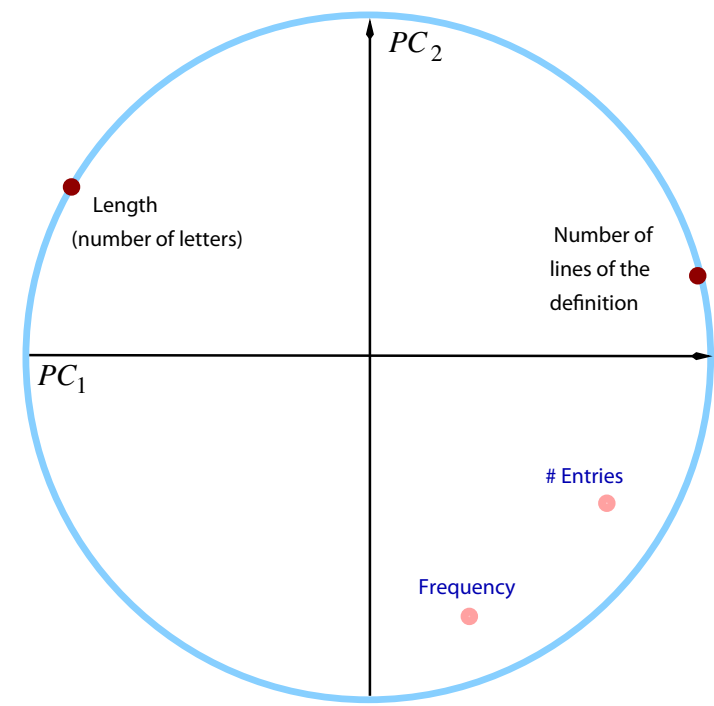

(b)

Figure 4: Circle of correlations and plot of the loadings of (a) the variables with principal components 1 and 2 , and (b) the variables and supplementary variables with principal components 1 and 2 . Note that the supplementary variables are not positioned on the unit circle.

\subsubsection{Plotting the correlations/loadings of the variables with the components}

The variables can be plotted as points in the component space using their loadings as coordinates. This representation differs from the plot of the observations: The observations are represented by their projections, but the variables are represented by their correlations. Recall that the sum of the squared loadings for a variable is equal to one. Remember, also, that a circle is defined as the set of points with the property that the sum of their squared coordinates is equal to a constant. As a consequence, when the data are perfectly represented by only two components, the sum of the squared loadings is equal to one, and therefore, in this case, the loadings will be positioned on a circle which is called the circle of correlations. When more than two components are needed to represent the data perfectly, the variables will be positioned inside the circle of correlations. The closer a variable is to the circle of correlations, the better we can reconstruct this variable from the first two components (and the more important it is to interpret these components); the closer to the center of the plot a variable is, the less important it is for the first two components.

Figure 4 shows the plot of the loadings of the variables on the components. Each variable is a point whose coordinates are given by the loadings on the principal components. 
Table 4: Supplementary variables for the example length of words and number of lines. "Frequency" is expressed as number of occurrences per 100,000 words, "\# Entries" is obtained by counting the number of entries for the word in the dictionary.

\begin{tabular}{lrr}
\hline & Frequency & \# Entries \\
\hline bag & 8 & 6 \\
across & 230 & 3 \\
on & 700 & 12 \\
insane & 1 & 2 \\
by & 500 & 7 \\
monastery & 1 & 1 \\
relief & 9 & 1 \\
slope & 2 & 6 \\
scoundrel & 1 & 1 \\
with & 700 & 5 \\
neither & 7 & 2 \\
pretentious & 1 & 1 \\
solid & 4 & 5 \\
this & 500 & 9 \\
for & 900 & 7 \\
therefore & 3 & 1 \\
generality & 1 & 1 \\
arise & 10 & 4 \\
blot & 1 & 4 \\
infectious & 1 & 2 \\
\hline
\end{tabular}

We can also use supplementary variables to enrich the interpretation. A supplementary variable should be measured for the same observations used for the analysis (for all of them or part of them, because we only need to compute a coefficient of correlation). After the analysis has been performed, the coefficients of correlation (i.e., the loadings) between the supplementary variables and the components are computed. Then the supplementary variables are displayed in the circle of correlations using the loadings as coordinates.

For example, we can add two supplementary variables to the word length and definition example. These data are shown in Table 4. A table of loadings for the supplementary variables can be computed from the coefficients of correlation between these variables and the components (see Table 5). Note that, contrary to the active variables, the squared loadings of the supplementary variables do not add up to 1 . 
Table 5: Loadings (i.e., coefficients of correlation) and squared loadings between supplementary variables and components.

\begin{tabular}{lrrrrrr}
\hline & \multicolumn{2}{c}{ Loadings } & & \multicolumn{2}{c}{ Squared Loadings } \\
\cline { 2 - 3 } \cline { 5 - 6 } Component & Frequency & \# Entries & & Frequency & \# Entries \\
\hline 1 & -.3012 & .6999 & & .0907 & .4899 \\
2 & -.7218 & -.4493 & & .5210 & .2019 \\
\cline { 5 - 6 }$\sum$ & & & & .6117 & .6918 \\
\hline
\end{tabular}

\section{Statistical inference: Evaluating the quality of the model}

\subsection{Fixed Effect Model}

The results of PCA so far correspond to a fixed effect model (i.e., the observations are considered to be the population of interest, and conclusions are limited to these specific observations). In this context, $\mathrm{PCA}_{\mathrm{C}}$ is descriptive and the amount of the variance of $\mathbf{X}$ explained by a component indicates its importance.

For a fixed effect model, the quality of the PCA model using the first $M$ components is obtained by first computing the estimated matrix, denoted $\widehat{\mathbf{X}}^{[M]}$, which is matrix $\mathbf{X}$ reconstituted with the first $M$ components. The formula for this estimation is obtained by combining Equations 1, 5, and 6 in order to obtain

$$
\mathbf{X}=\mathbf{F Q}^{\top}=\mathrm{XQQ}^{\top} \text {. }
$$

Then, the matrix $\widehat{\mathbf{X}}^{[M]}$ is built back using Equation 12 keeping only the first $M$ components:

$$
\begin{aligned}
\widehat{\mathbf{X}}^{[M]} & =\mathbf{P}^{[M]} \boldsymbol{\Delta}^{[M]} \mathbf{Q}^{[M] \top} \\
& =\mathbf{F}^{[M]} \mathbf{Q}^{[M] \top} \\
& =\mathbf{X Q}^{[M]} \mathbf{Q}^{[M] \top}
\end{aligned}
$$

where $\mathbf{P}^{[M]}, \boldsymbol{\Delta}^{[M]}$ and $\mathbf{Q}^{[M]}$ represent, respectively the matrices $\mathbf{P}, \boldsymbol{\Delta}$, and $\mathbf{Q}$ with only their first $M$ components. Note, incidently, that Equation 7 can be rewritten in the current context as:

$$
\mathbf{X}=\widehat{\mathbf{X}}^{[M]}+\mathbf{E}=\mathbf{F}^{[M]} \mathbf{Q}^{[M] \top}+\mathbf{E}
$$

(where $\mathbf{E}$ is the error matrix, which is equal to $\mathbf{X}-\widehat{\mathbf{X}}^{[M]}$ ).

To evaluate the quality of the reconstitution of $\mathbf{X}$ with $M$ components, we evaluate the similarity between $\mathbf{X}$ and $\widehat{\mathbf{X}}^{[M]}$. Several coefficients can be used for 
this task (see, e.g., Gower, 1971; Lingoes and Schönemann, 1974; Abdi, 2007c). The squared coefficient of correlation is sometimes used, as well as the $R_{V}$ coefficient (Dray, 2008; Abdi, 2007c). The most popular coefficient, however, is the residual sum of squares (RESS). It is computed as:

$$
\begin{aligned}
\operatorname{RESS}_{M} & =\left\|\mathbf{X}-\widehat{\mathbf{X}}^{[M]}\right\|^{2} \\
& =\operatorname{trace}\left\{\mathbf{E}^{\top} \mathbf{E}\right\} \\
& =\mathcal{I}-\sum_{\ell=1}^{M} \lambda_{\ell}
\end{aligned}
$$

where \|\| is the norm of $\mathbf{X}$ (i.e., the square root of the sum of all the squared elements of $\mathbf{X}$ ), and where the trace of a matrix is the sum of its diagonal elements. The smaller the value of RESs, the better the PCA model. For a fixed effect model, a larger $M$ gives a better estimation of $\widehat{\mathbf{X}}^{[M]}$. For a fixed effect model, the matrix $\mathbf{X}$ is always perfectly reconstituted with $L$ components (recall that $L$ is the rank of $\mathbf{X})$.

In addition, Equation 12 can be adapted to compute the estimation of the supplementary observations as

$$
\widehat{\mathbf{x}}_{\text {sup }}^{[M]}=\mathbf{x}_{\text {sup }} \mathbf{Q}^{[M]} \mathbf{Q}^{[M] \top} .
$$

\subsection{Random Effect Model}

In most applications, the set of observations represents a sample from a larger population. In this case, the goal is to estimate the value of new observations from this population. This corresponds to a random effect model. In order to estimate the generalization capacity of the PCA model, we cannot use standard parametric procedures. Therefore, the performance of the PCA model is evaluated using computer-based resampling techniques such as the bootstrap and cross-validation techniques where the data are separated into a learning and a testing set. A popular cross-validation technique is the jackknife (aka "leave one out" procedure). In the jackknife (Quenouille, 1956; Efron, 1982; Abdi and Williams, in press-c), each observation is dropped from the set in turn and the remaining observations constitute the learning set. The learning set is then used to estimate (using Equation 16) the left-out observation which constitutes the testing set. Using this procedure, each observation is estimated according to a random effect model. The predicted observations are then stored in a matrix denoted $\widetilde{\mathbf{X}}$.

The overall quality of the PCA random effect model using $M$ components is evaluated as the similarity between $\mathbf{X}$ and $\widetilde{\mathbf{X}}^{[M]}$. As with the fixed effect model, 
this can also be done with a squared coefficient of correlation or (better) with the $R_{V}$ coefficient. Similar to RESS, one can use the predicted residual sum of squares (PRESS). It is computed as:

$$
\operatorname{PRESS}_{M}=\left\|\mathbf{X}-\widetilde{\mathbf{X}}^{[M]}\right\|^{2} .
$$

The smaller the PRESS the better the quality of the estimation for a random model.

Contrary to what happens with the fixed effect model, the matrix $\mathbf{X}$ is not always perfectly reconstituted with all $L$ components. This is particularly the case when the number of variables is larger than the number of observations (a configuration known as the "small $N$ large $P$ " problem in the literature).

\subsection{How many components?}

Often, only the important information needs to be extracted from a data matrix. In this case, the problem is to figure out how many components need to be considered. This problem is still open, but there are some guidelines (see, e.g., Jackson, 1991; Jolliffe, 2002; Peres-Neto, Jackson, and Somers, 2005). A first procedure is to plot the eigenvalues according to their size (the so called "scree," see Cattell, 1966; Jolliffe, 2002) and to see if there is a point in this graph (often called an "elbow") such that the slope of the graph goes from "steep" to "flat" and to keep only the components which are before the elbow. This procedure, somewhat subjective, is called the scree or elbow test.

Another standard tradition is to keep only the components whose eigenvalue is larger than the average eigenvalue. Formally, this amount to keeping the $\ell$-th component if

$$
\lambda_{\ell}>\frac{1}{L} \sum_{\ell}^{L} \lambda_{\ell}=\frac{1}{L} \mathcal{I}
$$

(where $L$ is the rank of $\mathbf{X}$ ). For a correlation PCA, this rule boils down to the standard advice to "keep only the eigenvalues larger than 1" (see, e.g., Kaiser, 1961). However, this procedure can lead to ignoring important information (see O'Toole, Abdi, Deffenbacher, and Valentin, 1993, for an example of this problem).

\subsubsection{Random model}

As mentioned earlier, when using a random model, the quality of the prediction does not always increase with the number of components of the model. In fact, when the number of variables exceeds the number of observations, quality typically 
increases and then decreases. When the quality of the prediction decreases as the number of components increases this is an indication that the model is overfitting the data (i.e., the information in the learning set is not useful to fit the testing set). Therefore, it is important to determine the optimal number of components to keep when the goal is to generalize the conclusions of an analysis to new data.

A simple approach stops adding components when PRESs decreases. A more elaborated approach (see e.g., Geisser, 1974; Tennenhaus, 1998; Stone, 1974; Wold, 1995; Malinowski, 2002) begins by computing, for each component $\ell$, a quantity denoted $Q_{\ell}^{2}$ which is defined as:

$$
Q_{\ell}^{2}=1-\frac{\operatorname{PRESS}_{\ell}}{\operatorname{RESS}_{\ell-1}}
$$

with $\operatorname{PRESS}_{\ell}\left(\operatorname{RESS}_{\ell}\right)$ being the value of PRESS (RESS) for the $\ell$ th component (where $\operatorname{RESS}_{0}$ is equal to the total inertia). Only the components with $Q_{\ell}^{2}$ greater than or equal to an arbitrary critical value (usually $1-.95^{2}=.0975$ ) are kept (an alternative set of critical values sets the threshold to .05 when $I \leq 100$ and to 0 when $I>100$; see Tennenhaus, 1998).

Another approach - based on cross-validation - to decide upon the number of components to keep uses the index $W_{\ell}$ derived from Eastment and Krzanowski (1982) and Wold (1978). In contrast to $Q_{\ell}^{2}$, which depends on RESS and PRESS, the index $W_{\ell}$, depends only upon PRESS. It is computed for the $\ell$-th component as

$$
W_{\ell}=\frac{\operatorname{PRESS}_{\ell-1}-\operatorname{PRESS}_{\ell}}{\operatorname{PRESS}_{\ell}} \times \frac{d f_{\text {residual }, \ell}}{d f_{\ell}},
$$

where $\operatorname{PRESS}_{0}$ is the inertia of the data table, $d f_{\ell}$ is the number of degrees of freedom for the $\ell$-th component equal to

$$
d f_{\ell}=I+J-2 \ell,
$$

and $d f_{\text {residual, } \ell}$ is the residual number of degrees of freedom which is equal to the total number of degrees of freedom of the table [equal to $J(I-1)$ ] minus the number of degrees of freedom used by the previous components. The value of $d f_{\text {residual, } \ell}$ is obtained as

$$
d f_{\text {residual }, \ell}=J(I-1)-\sum_{k=1}^{\ell}(I+J-2 k)=J(I-1)-\ell(I+J-\ell-1) .
$$

Most of the time, $Q_{\ell}^{2}$ and $W_{\ell}$ will agree on the number of components to keep, but $W_{\ell}$ can give a more conservative estimate of the number of components to keep than $Q_{\ell}^{2}$. When $J$ is smaller than $I$, the value of both $Q_{L}^{2}$ and $W_{L}$ is meaningless because they both involve a division by zero. 


\subsection{Bootstraped confidence intervals}

After the number of components to keep has been determined, we can compute confidence intervals for the eigenvalues of $\widetilde{\mathbf{X}}$ using the bootstrap (Diaconis and Efron, 1983; Holmes, 1989; Efron and Tibshirani, 1993; Jackson, 1993, 1995; Mehlman, Sheperd, and Kelt, 1995). To use the bootstrap, we draw a large number of samples (e.g., 1,000 or 10,000) with replacement from the learning set. Each sample produces a set of eigenvalues. The whole set of eigenvalues can then be used to compute confidence intervals.

\section{Rotation}

After the number of components has been determined, and in order to facilitate the interpretation, the analysis often involves a rotation of the components that were retained (see, e.g., Abdi, 2003b, for more details). Two main types of rotation are used: orthogonal when the new axes are also orthogonal to each other, and oblique when the new axes are not required to be orthogonal. Because the rotations are always performed in a subspace, the new axes will always explain less inertia than the original components (which are computed to be optimal). However, the part of the inertia explained by the total subspace after rotation is the same as it was before rotation (only the partition of the inertia has changed). It is also important to note that because rotation always takes place in a subspace (i.e., the space of the retained components), the choice of this subspace strongly influences the result of the rotation. Therefore, it is strongly recommended to try several sizes for the subspace of the retained components in order to assess the robustness of the interpretation of the rotation. When performing a rotation, the term loadings almost always refer to the elements of matrix $\mathbf{Q}$. We will follow this tradition in this section.

\subsection{Orthogonal rotation}

An orthogonal rotation is specified by a rotation matrix, denoted $\mathbf{R}$, where the rows stand for the original factors and the columns for the new (rotated) factors. At the intersection of row $m$ and column $n$ we have the cosine of the angle between the original axis and the new one: $r_{m, n}=\cos \theta_{m, n}$. A rotation matrix has the important property of being orthonormal because it corresponds to a matrix of direction cosines and therefore $\mathbf{R}^{\top} \mathbf{R}=\mathbf{I}$. 
VARIMAX rotation, developed by Kaiser (1958), is the most popular rotation method. For VARIMAX a simple solution means that each component has a small number of large loadings and a large number of zero (or small) loadings. This simplifies interpretation because, after a VARIMAX rotation, each original variable tends to be associated with one (or a small number) of components, and each component represents only a small number of variables. In addition, the components can often be interpreted from the opposition of few variables with positive loadings to few variables with negative loadings. Formally VARIMAX searches for a linear combination of the original factors such that the variance of the squared loadings is maximized, which amounts to maximizing

$$
\nu=\sum\left(q_{j, \ell}^{2}-\bar{q}_{\ell}^{2}\right)^{2}
$$

with $q_{j, \ell}^{2}$ being the squared loading of the $j$ th variable of matrix $\mathbf{Q}$ on component $\ell$ and $\bar{q}_{\ell}^{2}$ being the mean of the squared loadings.

\subsection{Oblique Rotations}

With oblique rotations, the new axes are free to take any position in the component space, but the degree of correlation allowed among factors is small because two highly correlated components are better interpreted as only one factor. Oblique rotations, therefore, relax the orthogonality constraint in order to gain simplicity in the interpretation. They were strongly recommended by Thurstone (1947), but are used more rarely than their orthogonal counterparts.

For oblique rotations, the PROMAX rotation has the advantage of being fast and conceptually simple. The first step in PROMAX rotation defines the target matrix, almost always obtained as the result of a VARIMAX rotation whose entries are raised to some power (typically between 2 and 4 ) in order to force the structure of the loadings to become bipolar. The second step is obtained by computing a least square fit from the VARIMAX solution to the target matrix. PROMAX rotations are interpreted by looking at the correlations - regarded as loadings-between the rotated axes and the original variables. An interesting recent development of the concept of oblique rotation corresponds to the technique of independent component analysis (ICA) where the axes are computed in order to replace the notion of orthogonality by statistical independence (see Stone, 2004, for a tutorial).

\subsection{When and why using rotations}

The main reason for using rotation is to facilitate the interpretation. When the data follow a model (such as the psychometric model) stipulating 1) that each variable 
Table 6: An (artificial) example of PCA using a centered and normalized matrix. Five wines are described by seven variables (data from Abdi, in press).

\begin{tabular}{lrrrrrrr}
\hline & & $\begin{array}{r}\text { For } \\
\text { meat }\end{array}$ & $\begin{array}{r}\text { For } \\
\text { dessert }\end{array}$ & Price & Sugar & Alcohol & Acidity \\
\hline Wine 1 & 14 & 7 & 8 & 7 & 7 & 13 & 7 \\
Wine 2 & 10 & 7 & 6 & 4 & 3 & 14 & 7 \\
Wine 3 & 8 & 5 & 5 & 10 & 5 & 12 & 5 \\
Wine 4 & 2 & 4 & 7 & 16 & 7 & 11 & 3 \\
Wine 5 & 6 & 2 & 4 & 13 & 3 & 10 & 3 \\
\hline
\end{tabular}

load on only one factor and 2) that there is a clear difference in intensity between the relevant factors (whose eigenvalues are clearly larger than one) and the noise (represented by factors with eigenvalues clearly smaller than one), then the rotation is likely to provide a solution that is more reliable than the original solution. However if this model does not accurately represent the data, then rotation will make the solution less replicable and potentially harder to interpret because the mathematical properties of PCA have been lost.

\section{Examples}

\subsection{Correlation PCA}

Suppose that we have five wines described by the average ratings of a set of experts on their hedonic dimension, how much the wine goes with dessert, and how much the wine goes with meat. Each wine is also described by its price, its sugar and alcohol content, and its acidity. The data (from Abdi, 2003b, in press) are given in Table 6.

A PCA of this table extracts four factors (with eigenvalues of 4.76, 1.81, 0.35, and 0.07, respectively). Only two components have an eigenvalue larger than 1 and, together, these two components account for $94 \%$ of the inertia. The factor scores for the first two components are given in Table 7 and the corresponding map is displayed in Figure 5.

We can see from Figure 5 that the first component separates Wines 1 and 2 from Wines 4 and 5, while the second component separates Wines 2 and 5 from Wines 1 and 4. The examination of the values of the contributions and cosines, shown in Table 7, complements and refines this interpretation because the contributions suggest that Component 1 essentially contrasts Wines 1 and 2 with Wine 5 and that Component 2 essentially contrasts Wines 2 and 5 with Wine 4 . The cosines 
Table 7: PCA wine characteristics. Factor scores, contributions of the observations to the components, and squared cosines of the observations on principal components 1 and 2 . The positive important contributions are highlighted in light pink, and the negative important contributions are highlighted in red. For convenience, squared cosines and contributions have been multiplied by 100 and rounded.

\begin{tabular}{lrrrrrr}
\hline & $F_{1}$ & $F_{2}$ & $\operatorname{ctr}_{1}$ & $\operatorname{ctr}_{2}$ & $\cos _{1}^{2}$ & $\cos _{2}^{2}$ \\
\hline Wine 1 & -1.17 & -0.55 & 29 & 17 & 77 & 17 \\
Wine 2 & -1.04 & 0.61 & 23 & 21 & 69 & 24 \\
Wine 3 & 0.08 & 0.19 & 0 & 2 & 7 & 34 \\
Wine 4 & 0.89 & -0.86 & 17 & 41 & 50 & 46 \\
Wine 5 & 1.23 & 0.61 & 32 & 20 & 78 & 19 \\
\hline
\end{tabular}

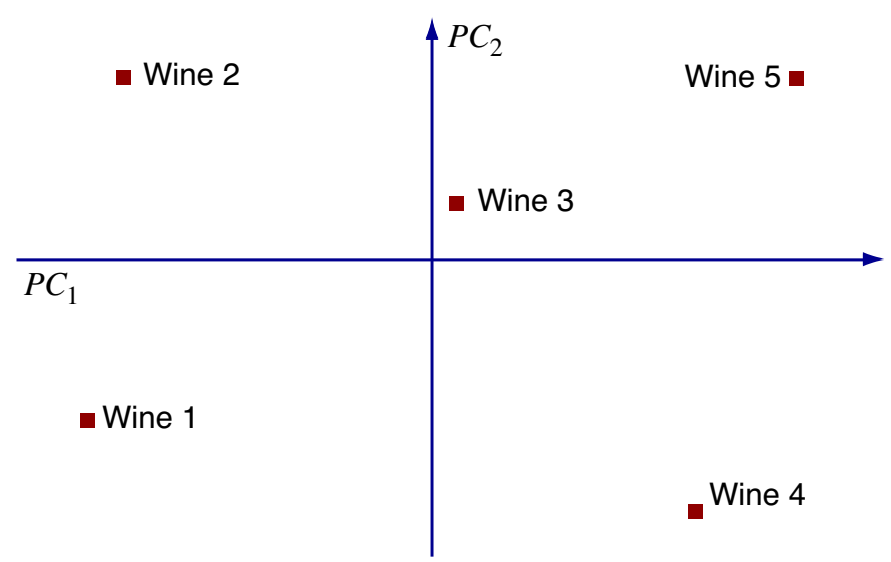

Figure 5: PCA wine characteristics. Factor scores of the observations plotted on the first 2 components. $\lambda_{1}=4.76$, $\tau_{1}=68 \% ; \lambda_{2}=1.81, \tau_{2}=26 \%$.

Table 8: PCA wine characteristics. Correlation of the variables with the first two components.

\begin{tabular}{rrrrrrrr}
\hline & & $\begin{array}{r}\text { For } \\
\text { meat }\end{array}$ & $\begin{array}{r}\text { For } \\
\text { dessert }\end{array}$ & Price & Sugar & Alcohol & Acidity \\
\hline PC 1 & -.87 & -.97 & -.58 & .91 & -.11 & -.96 & -.99 \\
PC 2 & .15 & -.15 & -.79 & -.42 & -.97 & .07 & .12 \\
\hline
\end{tabular}

show that Component 1 contributes highly to Wines 1 and 5, while Component 2 contributes most to Wine 4.

To find the variables that account for these differences, we examine the loadings of the variables on the first two components (see Table 9) and the circle of correlations (see Figure 6 and Table 8). From these, we see that the first component contrasts price with the wine's hedonic qualities, its acidity, its amount of alcohol, and how well it goes with meat (i.e., the wine tasters preferred inexpensive wines). The second component contrasts the wine's hedonic qualities, acidity and alcohol content with its sugar content and how well it goes with dessert. From 
Table 9: PCA wine characteristics. Loadings (i.e., $\mathrm{Q}$ matrix) of the variables on the first two components.

\begin{tabular}{rrrrrrrr}
\hline & & $\begin{array}{r}\text { For } \\
\text { meat }\end{array}$ & $\begin{array}{r}\text { For } \\
\text { dessert }\end{array}$ & Price & Sugar & Alcohol & Acidity \\
\hline PC 1 & -0.40 & -0.45 & -0.26 & 0.42 & -0.05 & -0.44 & -0.45 \\
PC 2 & 0.11 & -0.11 & -0.59 & -0.31 & -0.72 & 0.06 & 0.09 \\
\hline
\end{tabular}

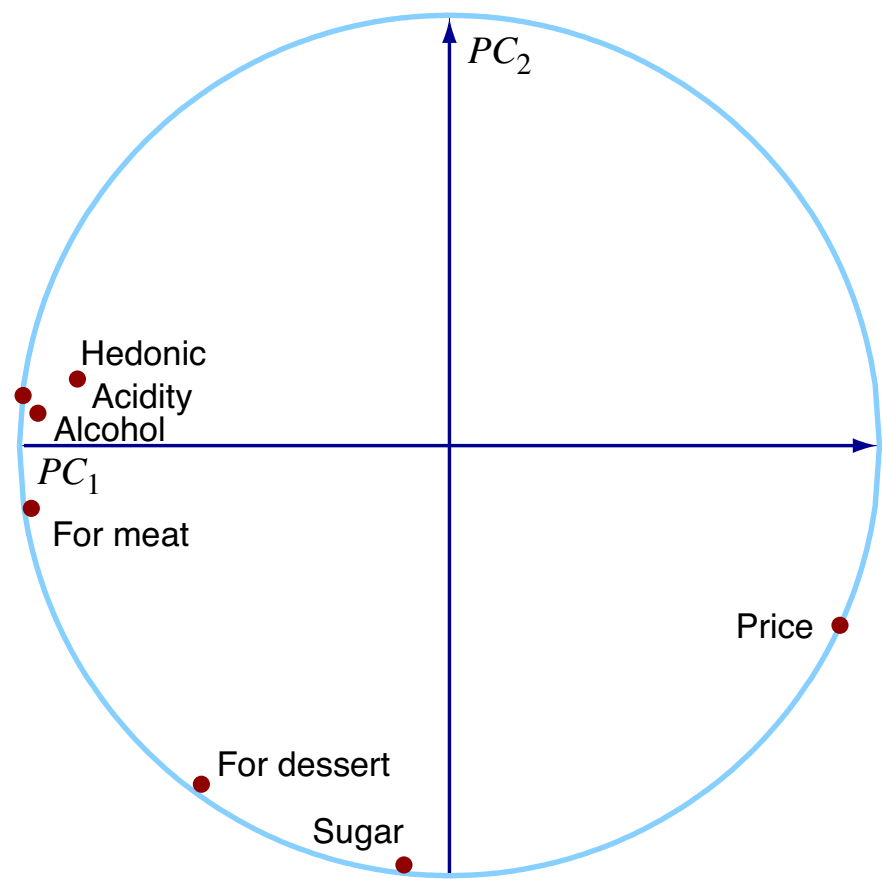

Figure 6: PCA wine characteristics. Correlation (and circle of correlations) of the variables with Components 1 and 2 . $\lambda_{1}=4.76, \tau_{1}=68 \% ; \lambda_{2}=1.81, \tau_{2}=26 \%$

this, it appears that the first component represents characteristics that are inversely correlated with a wine's price while the second component represents the wine's sweetness.

To strengthen the interpretation, we can apply a VARIMAX rotation, which gives a clockwise rotation of 15 degrees (corresponding to a cosine of .97). This gives the new set of rotated loadings shown in Table 10. The rotation procedure is illustrated in Figure 7. The improvement in the simplicity of the interpretation is marginal, maybe because the component structure of such a small data set is already very simple. The first dimension remains linked to price and the second dimension now appears more clearly as the dimension of sweetness. 
Table 10: PCA wine characteristics: Loadings (i.e., $\mathbf{Q}$ matrix), after VARIMAX rotation, of the variables on the first two components.

\begin{tabular}{rrrrrrrr}
\hline & & $\begin{array}{r}\text { For } \\
\text { meat }\end{array}$ & $\begin{array}{r}\text { For } \\
\text { dessert }\end{array}$ & Price & Sugar & Alcohol & Acidity \\
\hline PC 1 & -0.41 & -0.41 & -0.11 & 0.48 & 0.13 & -0.44 & -0.46 \\
PC 2 & 0.02 & -0.21 & -0.63 & -0.20 & -0.71 & -0.05 & -0.03 \\
\hline
\end{tabular}
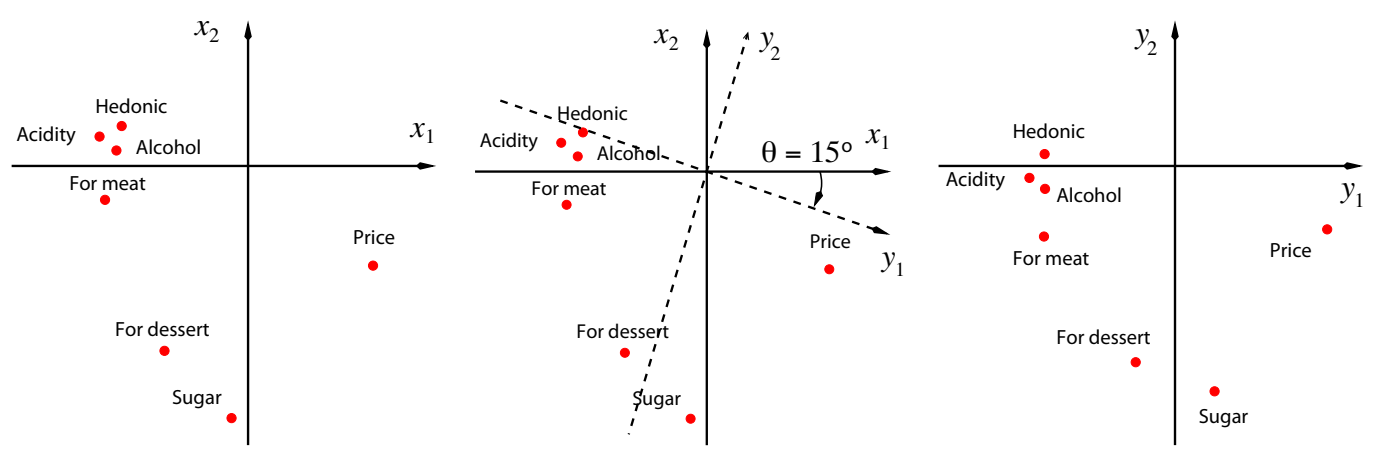

Figure 7: PCA wine characteristics: (a) Original loadings of the seven variables; (b) The loading of the seven variables showing the original axes and the new (rotated) axes derived from VARIMAX; (c) The loadings after VARIMAX rotation of the seven variables.

\subsection{Covariance PCA}

Here we use data from a survey performed in the 1950's in France (data from Lebart and Fénelon, 1975). The data table gives the average number of Francs spent on several categories of food products according to social class and the number of children per family. Because a Franc spent on one item has the same value as a Franc spent on another item, we want to keep the same unit of measurement for the complete space. Therefore we will perform a covariance PCA, rather than a correlation PCA. The data are shown in Table 11.

A PCA of the data table extracts 7 components (with eigenvalues of 3,023,141.24, 290,575.84, 68,795.23, 25,298.95, 22,992.25, 3,722.32, and 723.92, respectively). The first two components extract $96 \%$ of the inertia of the data table, and we will keep only these two components for further consideration (see also Table 14 for the choice of the number of components to keep). The factor scores for the first 2 components are given in Table 12 and the corresponding map is displayed in Figure 8.

We can see from Figure 8 that the first component separates the different social classes, while the second component reflects the number of children per family. This shows that buying patterns differ both by social class and by number of children per family. The contributions and cosines, given in Table 12, confirm this 
Table 11: Average number of Francs spent (per month) on different types of food according to social class and number of children (dataset from Lebart and Fénelon, 1975).

\begin{tabular}{|c|c|c|c|c|c|c|c|c|}
\hline & & \multicolumn{7}{|c|}{ Type of Food } \\
\hline & & Bread & Vegetables & Fruit & Meat & Poultry & Milk & Wine \\
\hline Blue Collar & 2 Children & 332 & 428 & 354 & 1437 & 526 & 247 & 427 \\
\hline White Collar & 2 Children & 293 & 559 & 388 & 1527 & 567 & 239 & 258 \\
\hline Upper Class & 2 Children & 372 & 767 & 562 & 1948 & 927 & 235 & 433 \\
\hline Blue Collar & 3 Children & 406 & 563 & 341 & 1507 & 544 & 324 & 407 \\
\hline White Collar & 3 Children & 386 & 608 & 396 & 1501 & 558 & 319 & 363 \\
\hline Upper Class & 3 Children & 438 & 843 & 689 & 2345 & 1148 & 243 & 341 \\
\hline Blue Collar & 4 Children & 534 & 660 & 367 & 1620 & 638 & 414 & 407 \\
\hline White Collar & 4 Children & 460 & 699 & 484 & 1856 & 762 & 400 & 416 \\
\hline Upper Class & 4 Children & 385 & 789 & 621 & 2366 & 1149 & 304 & 282 \\
\hline Blue Collar & 5 Children & 655 & 776 & 423 & 1848 & 759 & 495 & 486 \\
\hline White Collar & 5 Children & 584 & 995 & 548 & 2056 & 893 & 518 & 319 \\
\hline Upper Class & 5 Children & 515 & 1097 & 887 & 2630 & 1167 & 561 & 284 \\
\hline Mean & & 447 & 732 & 505 & 1887 & 803 & 358 & 369 \\
\hline$\hat{S}$ & & 107 & 189 & 165 & 396 & 250 & 117 & 72 \\
\hline
\end{tabular}

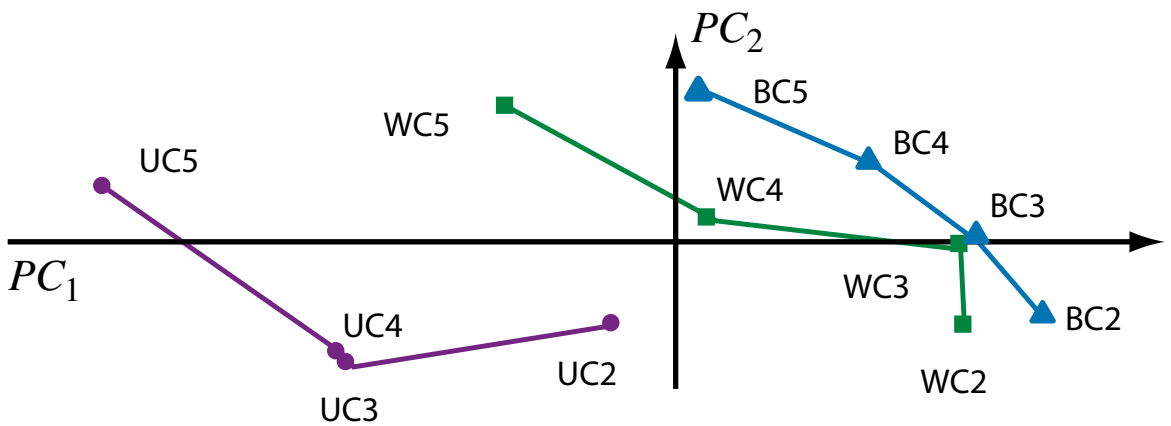

Figure 8: PCA example: Amount of Francs spent (per month) on food type by social class and number of children. Factor scores for principal components 1 and $2 . \lambda_{1}=3,023,141.24, \tau_{1}=88 \% ; \lambda_{2}=290,575.84, \tau_{2}=8 \%$. BC $=$ blue collar; WC $=$ white collar; UC $=$ upper class; $2=2$ children; $3=3$ children; $4=4$ children; $5=5$ children.

interpretation. The values of the contributions of the observations to the components indicate that Component 1 contrasts blue collar families with 3 children to upper class families with 3 or more children whereas Component 2 contrasts blue and white collar families with 5 children to upper class families with 3 and 4 children. In addition, the cosines between the components and the variables show that Component 1 contributes to the pattern of food spending seen by the blue collar and white collar families with 2 and 3 children and to the upper class families with 3 or more children while Component 2 contributes to the pattern of food spending by blue collar families with 5 children. 
Table 12: PCA example. Amount of Francs spent (per month) by food type, social class, and number of children. Factor scores, contributions of the observations to the components, and squared cosines of the observations on principal components 1 and 2. The positive important contributions are highlighted in light pink, and the negative important contributions are highlighted in red. For convenience, squared cosines and contributions have been multiplied by 100 and rounded.

\begin{tabular}{llrrrrrr}
\hline & & $F_{1}$ & $F_{2}$ & $\operatorname{ctr}_{1}$ & $\operatorname{ctr}_{2}$ & $\cos _{1}^{2}$ & $\cos _{2}^{2}$ \\
\hline Blue Collar & 2 Children & 635.05 & -120.89 & 13 & 5 & 95 & 3 \\
White Collar & 2 Children & 488.56 & -142.33 & 8 & 7 & 86 & 7 \\
Upper Class & 2 Children & -112.03 & -139.75 & 0 & 7 & 26 & 40 \\
Blue Collar & 3 Children & 520.01 & 12.05 & 9 & 0 & 100 & 0 \\
White Collar & 3 Children & 485.94 & 1.17 & 8 & 0 & 98 & 0 \\
Upper Class & 3 Children & -588.17 & -188.44 & 11 & 12 & 89 & 9 \\
Blue Collar & 4 Children & 333.95 & 144.54 & 4 & 7 & 83 & 15 \\
White Collar & 4 Children & 57.51 & 42.86 & 0 & 1 & 40 & 22 \\
Upper Class & 4 Children & -571.32 & -206.76 & 11 & 15 & 86 & 11 \\
Blue Collar & 5 Children & 39.38 & 264.47 & 0 & 24 & 2 & 79 \\
White Collar & 5 Children & -296.04 & 235.92 & 3 & 19 & 57 & 36 \\
Upper Class & 5 Children & -992.83 & 97.15 & 33 & 3 & 97 & 1 \\
\hline
\end{tabular}

Table 13: PCA example: Amount of Francs spent (per month) on food type by social class and number of children. Squared loadings of the variables on Components 1 and 2 .

\begin{tabular}{rrrrrrrr}
\hline & Bread & Vegetables & Fruit & Meat & Poultry & Milk & Wine \\
\hline PC 1 & 0.01 & 0.33 & 0.16 & 0.01 & 0.03 & 0.45 & 0.00 \\
PC 2 & 0.11 & 0.17 & 0.09 & 0.37 & 0.18 & 0.03 & 0.06 \\
\hline
\end{tabular}

To find the variables that account for these differences, we refer to the squared loadings of the variables on the 2 components (Table 13) and to the circle of correlations (see Figure 9). From these, we see that the first component contrasts the amount spent on wine with all other food purchases, while the second component contrasts the purchase of milk and bread with meat, fruit, and poultry. This indicates that wealthier families spend more money on meat, poultry and fruit when they have more children, while white and blue collar families spend more money on bread and milk when they have more children. In addition, the number of children in upper class families seems inversely correlated with the consumption of wine (i.e., wealthy families with 4 or 5 children consume less wine than all other types of families). This curious effect is understandable when placed in the context of the French culture of the 1950s, in which wealthier families with many children tended to be rather religious and therefore less inclined to indulge in the consumption of wine.

Recall that the first two components account for $96 \%$ of the total inertia [i.e., $\left.\left(\lambda_{1}+\lambda_{2}\right) / \mathcal{I}=(3,023,141.24+290,575.84) / 3,435,249.75=.96\right]$. From Table 14 we find that $\mathrm{RESS}_{2}$ is equal to $4 \%$ and this value represents the error when $\widehat{\mathrm{X}}$ is estimated from Components 1 and 2 together. This means that for a fixed effect 


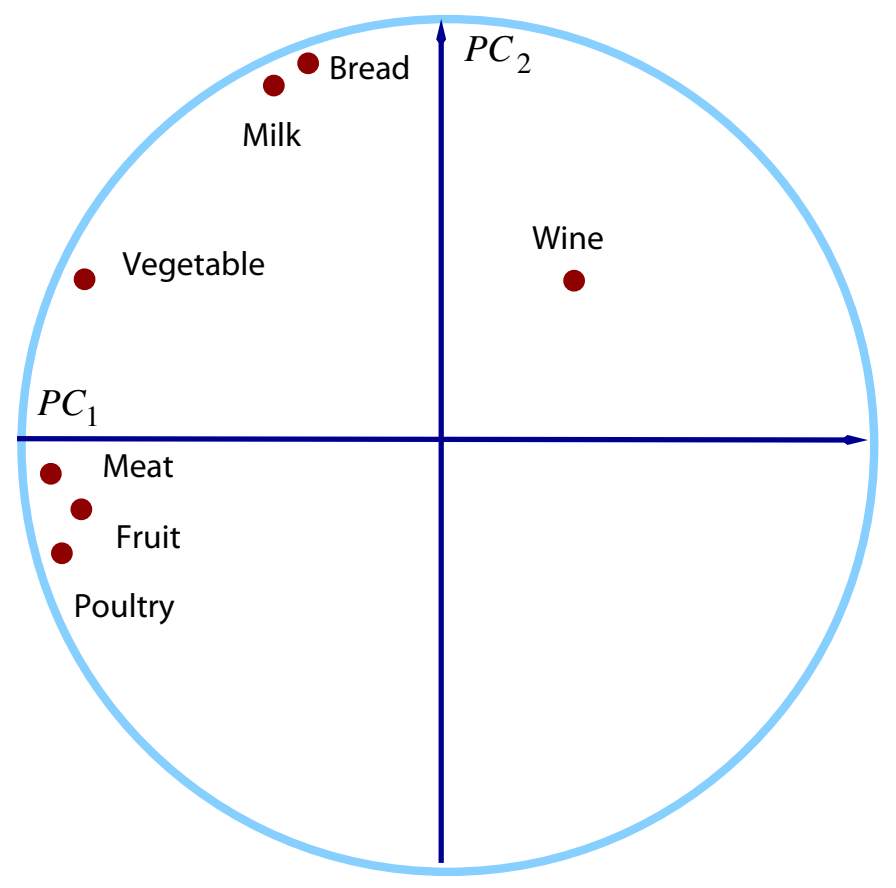

Figure 9: PCA example: Amount of Francs spent (per month) on food type by social class and number of children. Correlations (and circle of correlations) of the variables with components 1 and $2 . \lambda_{1}=3,023,141.24, \tau_{1}=88 \%$; $\lambda_{2}=290,575.84, \tau_{2}=8 \%$.

model, a 2-component solution represents $\mathbf{X}$ well. $\mathrm{PRESS}_{2}$, the error of estimation using a random effect model with two components, is equal to $8 \%$ and this value indicates that $\widetilde{\mathbf{X}}$ represents $\mathbf{X}$ adequately. Together, the values of RESS and PRESS suggest that only the first two components should be kept.

To confirm the number of components to keep, we look at $Q^{2}$ and $W$. The $Q^{2}$ values of .82 and .37 for Components 1 and 2 both exceed the critical value of .095, indicating that both components should be kept. Note that a negative $Q^{2}$ value suggests that a component should not be kept. In contrast, the $W$ values of 1.31 and .45 for the first two components suggest that only the first component should be kept because only $W_{1}$ is greater than 1 . 


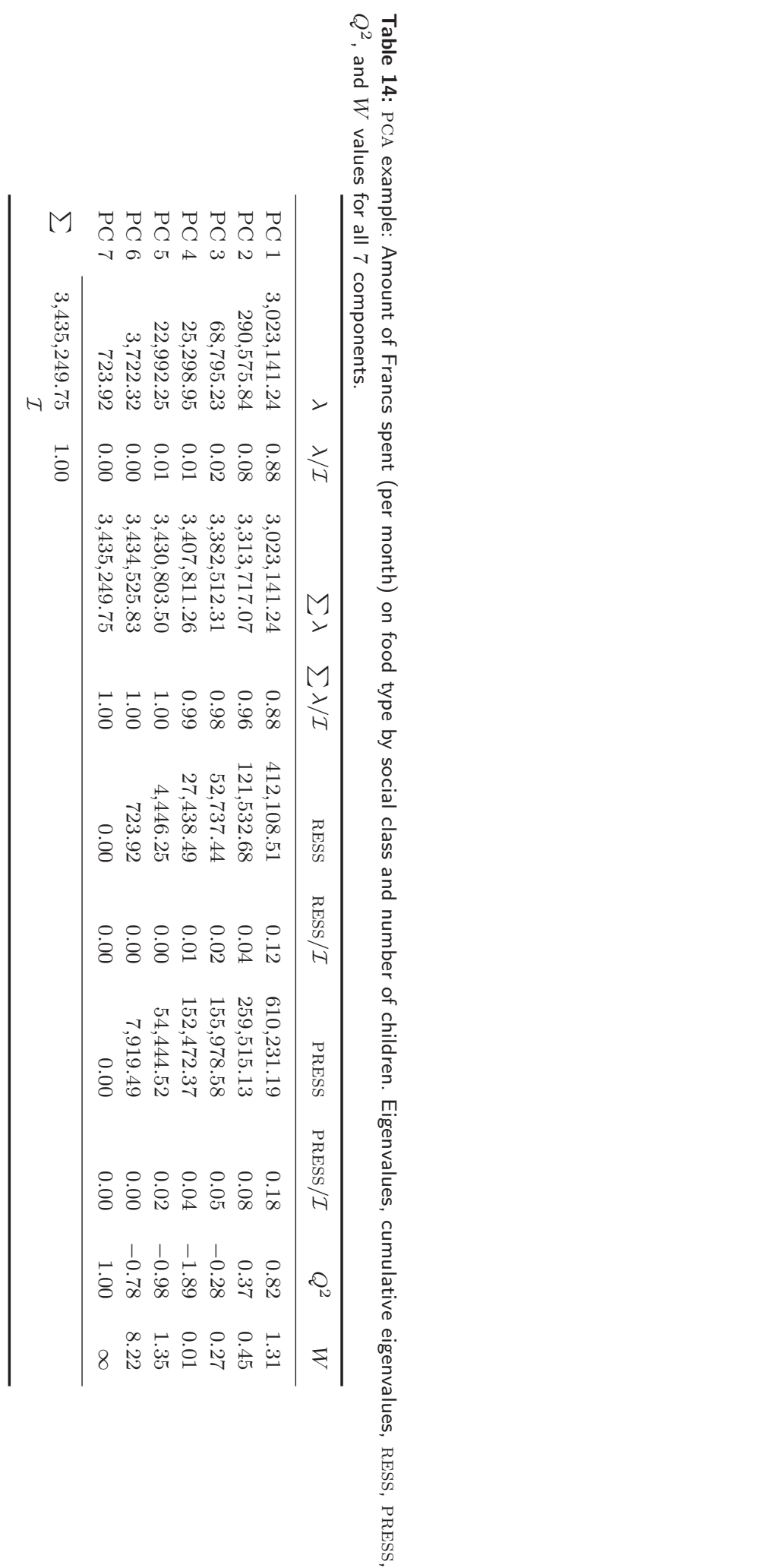




\section{Some extensions of PCA}

\subsection{Correspondence Analysis}

Correspondence analysis (CA; see Benzécri, 1973; Greenacre, 1984, 2007; Abdi and Valentin, 2007a; Hwang, Tomiuk, and Takane, in press; Abdi and Williams, in press-b) is an adaptation of PCA tailored to handle nominal variables. It can be interpreted as a particular case of generalized PCA for which we take into account masses (for the rows) and weights (for the columns). CA analyzes a contingency table and provides factor scores for both the rows and the columns of the contingency table. In correspondence analysis, the inertia of the contingency table is proportional to the $\chi^{2}$ which can be computed to test the independence of the rows and the columns of this table. Therefore the factor scores in CA decompose this independence $\chi^{2}$ into orthogonal components (in the CA tradition, these components are often called factors rather than components, here, for coherence, we keep the name component for both PCA and CA).

\subsubsection{Notations}

The $I \times J$ contingency table to be analyzed is denoted $\mathbf{X}$. CA will provide two sets of factor scores: one for the rows and one for the columns. These factor scores are, in general, scaled such that their inertia is equal to their eigenvalue (some versions of CA compute row or column factor scores normalized to unity). The grand total of the table is noted $N$.

\subsubsection{Computations}

The first step of the analysis is to compute the probability matrix $\mathbf{Z}=N^{-1} \mathbf{X}$. We denote $\mathbf{r}$ the vector of the row totals of $\mathbf{Z}$, (i.e., $\mathbf{r}=\mathbf{Z} \mathbf{1}$, with $\mathbf{1}$ being a conformable vector of 1's), $\mathbf{c}$ the vector of the columns totals, and $\mathbf{D}_{\mathbf{c}}=\operatorname{diag}\{\mathbf{c}\}$, $\mathbf{D}_{\mathbf{r}}=\operatorname{diag}\{\mathbf{r}\}$. The factor scores are obtained from the following generalized singular value decomposition (see Appendix B):

$$
\left(\mathbf{Z}-\mathbf{r c}^{\top}\right)=\widetilde{\mathbf{P}} \widetilde{\Delta} \widetilde{\mathbf{Q}}^{\top} \text { with } \widetilde{\mathbf{P}}^{\top} \mathbf{D}_{\mathbf{r}}^{-1} \widetilde{\mathbf{P}}=\widetilde{\mathbf{Q}}^{\top} \mathbf{D}_{\mathbf{c}}^{-1} \widetilde{\mathbf{Q}}=\mathbf{I}
$$

The row and (respectively) column factor scores are obtained as

$$
\mathbf{F}=\mathbf{D}_{\mathbf{r}}^{-1} \widetilde{\mathbf{P}} \widetilde{\Delta} \quad \text { and } \quad \mathrm{G}=\mathrm{D}_{\mathrm{c}}^{-1} \widetilde{\mathbf{Q}} \widetilde{\Delta}
$$

In $\mathrm{CA}$, the rows and the columns of the table have a similar role and therefore we have contributions and cosines for both sets. These are obtained in a similar way 
as for standard PCA, but the computations of the contributions need to integrate the values of the masses (i.e., the elements of $\mathbf{r}$ ) and weights (i.e., the elements of c). Specifically, the contribution of row $i$ to component $\ell$ and of column $j$ to component $\ell$ are obtained respectively as:

$$
\operatorname{ctr}_{i, \ell}=\frac{r_{i} f_{i, \ell}^{2}}{\lambda_{\ell}} \quad \text { and } \quad \operatorname{ctr}_{j, \ell}=\frac{c_{j} g_{j, \ell}^{2}}{\lambda_{\ell}}
$$

(with $r_{i}$ begin the $i$ th element of $\mathbf{r}$ and $c_{j}$ being the $j$ th element of $\mathbf{c}$ ). As for standard PCA, contributions help locating the observations or variables important for a given component.

The vector of the squared $\left(\chi^{2}\right)$ distance from the rows and columns to their respective barycenter are obtained as

$$
\mathbf{d}_{\mathbf{r}}=\operatorname{diag}\left\{\mathbf{F F}^{\top}\right\} \quad \text { and } \quad \mathbf{d}_{\mathbf{c}}=\operatorname{diag}\left\{\mathbf{G G}^{\top}\right\} .
$$

As for PCA, the total inertia in $\mathrm{CA}$ is equal to the sum of the eigenvalues. By contrast with PCA, the total inertia can also be computed equivalently as the weighted sum of the squared distances of the rows or the columns to their respective barycenter. Formally, the inertia can be computed as:

$$
\mathcal{I}=\sum_{l}^{L} \lambda_{\ell}=\mathbf{r}^{\top} \mathbf{d}_{\mathbf{r}}=\mathbf{c}^{\top} \mathbf{d}_{\mathbf{c}} .
$$

The squared cosine between row $i$ and component $\ell$ and column $j$ and component $\ell$ are obtained respectively as:

$$
\cos _{i, \ell}^{2}=\frac{f_{i, \ell}^{2}}{d_{r, i}^{2}} \quad \text { and } \quad \cos _{j, \ell}^{2}=\frac{g_{j, \ell}^{2}}{d_{c, j}^{2}} .
$$

(with $d_{r, i}^{2}$, and $d_{c, j}^{2}$, being respectively the $i$-th element of $\mathbf{d}_{\mathbf{r}}$ and the $j$-th element of $\mathbf{d}_{\mathbf{c}}$ ). Just like for PCA, squared cosines help locating the components important for a given observation or variable.

And just like for PCA, supplementary or illustrative elements can be projected onto the components, but the $\mathrm{CA}$ formula needs to take into account masses and weights. The projection formula, is called the transition formula and it is specific to correspondence analysis. Specifically, let $\mathbf{i}_{\text {sup }}^{\top}$ being an illustrative row and $\mathbf{j}_{\text {sup }}$ being an illustrative column to be projected (note that in CA, prior to projection, a illustrative row or column is re-scaled such that its sum is equal to one). Their coordinates of the illustrative rows (denoted $\mathbf{f}_{\text {sup }}$ ) and column (denoted $\mathbf{g}_{\text {sup }}$ ) are obtained as:

$$
\mathbf{f}_{\text {sup }}=\left(\mathbf{i}_{\text {sup }}^{\top} \mathbf{1}\right)^{-1} \mathbf{i}_{\text {sup }}^{\top} \mathbf{G} \widetilde{\Delta}^{-1} \text { and } \mathbf{g}_{\text {sup }}=\left(\mathbf{j}_{\text {sup }}^{\top} \mathbf{1}\right)^{-1} \mathbf{j}_{\text {sup }}^{\top} \mathbf{F} \widetilde{\Delta}^{-1}
$$


Table 15: The punctuation marks of six French writers (from Brunet, 1989). The column labelled $x_{i+}$ gives the total number of punctuation marks used by each author. $N$ is the grand total of the data table. The vector of mass for the rows, $\mathbf{r}$, is the proportion of punctuation marks used by each author $\left(r_{i}=x_{i+} / N\right)$. The row labelled $x_{+j}$ gives the total number of times each punctuation mark was used. The centroid row, $\mathbf{c}^{\top}$, gives the proportion of each punctuation mark in the sample $\left(c_{j}=x_{+j} / N\right)$.

\begin{tabular}{lrrrrr}
\hline Author's name & Period & Comma & Other & $x_{i+}$ & $\mathbf{r}$ \\
\hline Rousseau & 7,836 & 13,112 & 6,026 & 26,974 & .0189 \\
Chateaubriand & 53,655 & 102,383 & 42,413 & 198,451 & .1393 \\
Hugo & 115,615 & 184,541 & 59,226 & 359,382 & .2522 \\
Zola & 161,926 & 340,479 & 62,754 & 565,159 & .3966 \\
Proust & 38,177 & 105,101 & 12,670 & 155,948 & .1094 \\
Giraudoux & 46,371 & 58,367 & 14,299 & 119,037 & .0835 \\
\hline$x_{+j}$ & 423,580 & 803,983 & 197,388 & $N=142,4951$ & 1.0000 \\
$\mathbf{c}^{\top}$ & .2973 & .5642 & .1385 & & \\
\hline
\end{tabular}

[note that the scalar terms $\left(\mathbf{i}_{\text {sup }}^{\top} \mathbf{1}\right)^{-1}$ and $\left(\mathbf{j}_{\text {sup }}^{\top} \mathbf{1}\right)^{-1}$ are used to ensure that the sum of the elements of $\mathbf{i}_{\text {sup }}$ or $\mathbf{j}_{\text {sup }}$ is equal to one, if this is already the case, these terms are superfluous].

\subsubsection{Example}

For this example, we use a contingency table that gives the number of punctuation marks used by the French writers Rousseau, Chateaubriand, Hugo, Zola, Proust, and Giraudoux (data from Brunet, 1989). This table indicates how often each writer used the period, the comma, and all other punctuation marks combined (i.e., interrogation mark, exclamation mark, colon, and semi-colon). The data are shown in Table 15.

A CA of the punctuation table extracts two components which together account for $100 \%$ of the inertia (with eigenvalues of .0178 and .0056, respectively). The factor scores of the observations (rows) and variables (columns) are shown in Tables 16 and the corresponding map is displayed in Figure 10.

We can see from Figure 10 that the first component separates Proust and Zola's pattern of punctuation from the pattern of punctuation of the other 4 authors, with Chateaubriand, Proust and Zola contributing most to the component. The squared cosines show that the first component accounts for all of Zola's pattern of punctuation (see Table 16).

The second component separates Giraudoux's pattern of punctuation from that of the other authors. Giraudoux also has the highest contribution indicating that Giraudoux's pattern of punctuation is important for the second component. In ad- 
Table 16: CA punctuation. Factor scores, contributions, mass, mass $\times$ squared factor scores, inertia to barycenter, and squared cosines for the rows. The positive important contributions are highlighted in light pink, and the negative important contributions are highlighted in red. For convenience, squared cosines and contributions have been multiplied by 100 and rounded.

\begin{tabular}{|c|c|c|c|c|c|c|c|c|c|c|}
\hline & $F_{1}$ & $F_{2}$ & $\operatorname{ctr}_{1}$ & $\operatorname{ctr}_{2}$ & $r_{i}$ & $\begin{array}{r}r_{i} \times \\
F_{1}^{2}\end{array}$ & $\begin{array}{r}r_{i} \times \\
F_{2}^{2}\end{array}$ & $\begin{array}{c}r_{i} \times \\
d_{r, i}^{2}\end{array}$ & $\cos _{1}^{2}$ & $\cos _{2}^{2}$ \\
\hline Rousseau & -0.24 & -0.07 & 6 & 2 & .0189 & .0011 & .0001 & .0012 & 91 & 9 \\
\hline Chateaubriand & -0.19 & -0.11 & 28 & 29 & .1393 & .0050 & .0016 & .0066 & 76 & 24 \\
\hline Hugo & -0.10 & 0.03 & 15 & 4 & .2522 & .0027 & .0002 & .0029 & 92 & 8 \\
\hline Zola & 0.09 & -0.00 & 19 & 0 & .3966 & .0033 & .0000 & .0033 & 100 & 0 \\
\hline Proust & 0.22 & -0.06 & 31 & 8 & .1094 & .0055 & .0004 & .0059 & 93 & 7 \\
\hline Giraudoux & -0.05 & 0.20 & 1 & 58 & .0835 & .0002 & .0032 & .0034 & 6 & 94 \\
\hline$\sum$ & - & - & 100 & 100 & - & $\begin{array}{r}.0178 \\
\lambda_{1} \\
76 \% \\
\tau_{1}\end{array}$ & $\begin{array}{r}.0056 \\
\lambda_{2} \\
24 \% \\
\tau_{2}\end{array}$ & $\begin{array}{r}.0234 \\
\mathcal{I}\end{array}$ & & \\
\hline
\end{tabular}

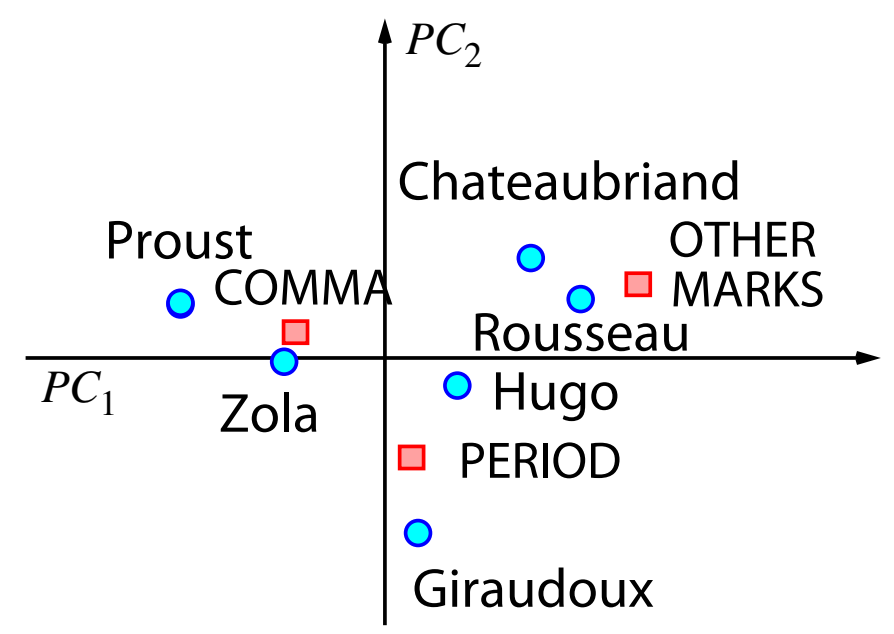

Figure 10: CA punctuation. The projections of the rows and the columns are displayed in the same map. $\lambda_{1}=.0178$, $\tau_{1}=76.16 ; \lambda_{2}=.0056, \tau_{2}=23.84$

dition, for Giraudoux the highest squared cosine (94\%), is obtained for Component 2. This shows that the second component is essential to understand Giraudoux's pattern of punctuation (see Table 16).

In contrast with PCA, the variables (columns) in CA are interpreted identically to the rows. The factor scores for the variables (columns) are shown in Table 17 and the corresponding map is displayed in the same map as the observations shown in Figure 10. 
Table 17: CA punctuation. Factor scores, contributions, mass, mass $\times$ squared factor scores, inertia to barycenter, and squared cosines for the columns. The positive important contributions are highlighted in light pink, and the negative important contributions are highlighted in red. For convenience, squared cosines and contributions have been multiplied by 100 and rounded.

\begin{tabular}{|c|c|c|c|c|c|c|c|c|c|c|}
\hline & $F_{1}$ & $F_{2}$ & $\operatorname{ctr}_{1}$ & $\operatorname{ctr}_{2}$ & $c_{j}$ & $\begin{array}{c}c_{j} \times \\
F_{1}^{2}\end{array}$ & $\begin{array}{r}c_{j} \times \\
F_{2}^{2}\end{array}$ & $\begin{array}{l}c_{j} \times \\
d_{c, j}^{2}\end{array}$ & $\cos _{1}^{2}$ & $\cos _{2}^{2}$ \\
\hline Period & -0.05 & 0.11 & 4 & 66 & .2973 & .0007 & .0037 & .0044 & 16 & 84 \\
\hline Comma & 0.10 & -0.04 & 30 & 14 & .5642 & .0053 & .0008 & .0061 & 88 & 12 \\
\hline Other & -0.29 & -0.09 & 66 & 20 & .1385 & .0118 & .0011 & .0129 & 91 & 9 \\
\hline \multirow[t]{4}{*}{$\sum$} & - & - & 100 & 100 & - & .0178 & .0056 & .0234 & & \\
\hline & & & & & & $\lambda_{1}$ & & $\mathcal{I}$ & & \\
\hline & & & & & & $76 \%$ & $24 \%$ & & & \\
\hline & & & & & & $\tau_{1}$ & $\tau_{2}$ & & & \\
\hline
\end{tabular}

From Figure 10 we can see that the first component also separates the comma from the "others" punctuation marks. This is supported by the high contributions of "others" and comma to the component. The cosines also support this interpretation because the first component accounts for $88 \%$ of the use of the comma and $91 \%$ of the use of the "others" punctuation marks (see Table 17).

The second component separates the period from both the comma and the "other" punctuation marks. This is supported by the period's high contribution to the second component and the component's contribution to the use of the period (see Table 17).

Together, the pattern of distribution of the points representing the authors and the punctuation marks suggests that some of the differences in the authors' respective styles can be attributed to differences in their use of punctuation. Specifically, Zola's œuvre is characterized by his larger than average use of the comma, while Chateaubriand's is characterized by his larger than average use of other types of punctuation marks than the period and the comma. In addition, Giraudoux's œuvre is characterized by a larger than average use of the period.

\subsection{Multiple factor analysis}

Multiple factor analysis (MFA; see Escofier and Pagès, 1990, 1994; Abdi and Valentin, $2007 \mathrm{~b}$ ) is used to analyze a set of observations described by several groups of variables. The number of variables in each group may differ and the nature of the variables (nominal or quantitative) can vary from one group to the other but the variables should be of the same nature in a given group. The analysis derives an 
integrated picture of the observations and of the relationships between the groups of variables.

\subsubsection{Notations}

The data consists of $T$ data sets. Each data set is called a subtable. Each subtable is an $I \times{ }_{[t]} J$ rectangular data matrix denoted ${ }_{[t]} \mathbf{Y}$, where $I$ is the number of observations and ${ }_{[t]} J$ the number of variables of the $t$-th subtable. The total number of variables is equal to $J$, with:

$$
J=\sum_{t}[t] J
$$

Each subtable is preprocessed (e.g., centered and normalized) and the preprocessed data matrices actually used in the analysis are denoted ${ }_{[t]} \mathbf{X}$.

The $\ell$-th eigenvalue of the $t$-th subtable is denoted $[t] \varrho_{\ell}$. The $\ell$-th singular value of the $t$-th subtable is denoted ${ }_{[t]} \varphi_{\ell}$.

\subsubsection{Computations}

The goal of MFA is to integrate different groups of variables (i.e., different subtables) describing the same observations. In order to do so, the first step is to make these subtables comparable. Such a step is needed because the straightforward analysis obtained by concatenating all variables would be dominated by the subtable with the strongest structure (which would be the subtable with the largest first singular value). In order to make the subtables comparable, we need to normalize them. To normalize a subtable, we first compute a PCA for this subtable. The first singular value (i.e., the square root of the first eigenvalue) is the normalizing factor which is used to divide the elements of this subtable. So, formally, The normalized subtables are computed as:

$$
{ }_{[t]} \mathbf{Z}=\frac{1}{\sqrt{[t] \varrho_{1}}} \times{ }_{[t]} \mathbf{X}=\frac{1}{[t] \varphi_{1}} \times{ }_{[t]} \mathbf{X} .
$$

The normalized subtables are concatenated into an $I \times J$ matrix called the global data matrix denoted $\mathbf{Z}$. A PCA is then run on $\mathbf{Z}$ to get a global solution. Note that because the subtables have previously been centered and normalized with their first singular value, $\mathbf{Z}$ is centered but it is not normalized (i.e., columns from different subtables have, in general, different norms). 
To find out how each subtable performs relative to the global solution, each subtable (i.e., each ${ }_{[t]} \mathbf{X}$ ) is projected into the global space as a supplementary element.

As in standard PCA, variable loadings are correlations between original variables and global factor scores. To find the relationship between the variables from each of the subtables and the global solution we compute loadings (i.e., correlations) between the components of each subtable and the components of the global analysis.

\subsubsection{Example}

Suppose that three experts were asked to rate 6 wines aged in two different kinds of oak barrel from the same harvest of Pinot Noir (example from Abdi and Valentin, 2007b). Wines 1, 5, and 6 were aged with a first type of oak, and Wines 2, 3, and 4 with a second type of oak. Each expert was asked to choose from 2 to 5 variables to describe the wines. For each wine, the expert rated the intensity of his/her variables on a 9-point scale. The data consist of $T=3$ subtables, which are presented in Table 18.

The PCA-S on each of the three subtables extracted eigenvalues of ${ }_{1} \varrho_{1}=2.86$, ${ }_{2} \varrho_{1}=3.65$, and ${ }_{3} \varrho_{1}=2.50$ with singular values of ${ }_{1} \varphi_{1}=1.69,{ }_{2} \varphi_{1}=1.91$, and ${ }_{3} \varphi_{1}=1.58$, respectively.

Following normalization and concatenation of the subtables, the global PCA extracted 5 components (with eigenvalues of $2.83,0.36,0.11,0.03$, and 0.01 ). The first 2 components explain $95 \%$ of the inertia. The factor scores for the first 2 components of the global analysis are given in Table 20 and the corresponding map is displayed in Figure 11a.

We can see from Figure 11 that the first component separates the first type of oak (wines 1, 5, and 6) from the second oak type (wines 2, 3, and 4).

In addition to examining the placement of the wines, we wanted to see how each expert's ratings fit into the global PCA space. We achieved this by projecting the data set of each expert as a supplementary element (see Abdi, 2007c, for details of the procedure). The factor scores are shown in Table 20. The experts' placement in the global map is shown in Figure 11b. Note that the position of each wine in the global analysis is the center of gravity of its position for the experts. The projection of the experts shows that Expert 3's ratings differ from those of the other two experts. 


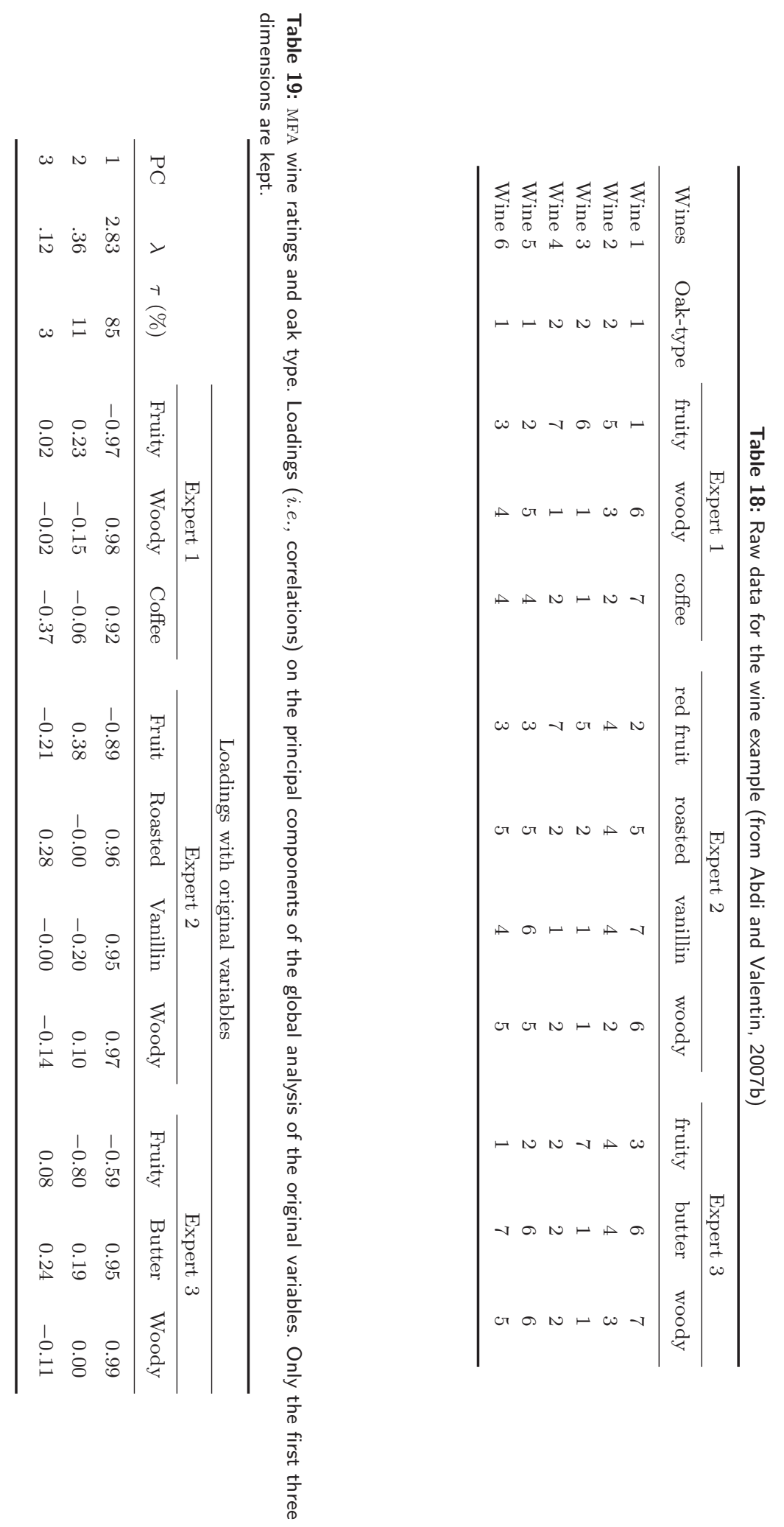


Table 20: MFA wine ratings and oak type. Factor scores for the global analysis, Expert 1, Expert 2, and Expert 3 for the first 2 components.

\begin{tabular}{|c|c|c|c|c|c|c|c|c|}
\hline & \multicolumn{2}{|c|}{ Global } & \multicolumn{2}{|c|}{ Expert $1_{\text {sup }}$} & \multicolumn{2}{|c|}{ Expert $2_{\text {sup }}$} & \multicolumn{2}{|c|}{ Expert $3_{\text {sup }}$} \\
\hline & $F_{1}$ & $F_{2}$ & ${ }_{[1]} F_{1}$ & ${ }_{[1]} F_{2}$ & ${ }_{[2]} F_{1}$ & ${ }_{[2]} F_{2}$ & ${ }_{[3]} F_{1}$ & ${ }_{[3]}{ }^{F_{2}}$ \\
\hline Wine 1 & 2.18 & -0.51 & 2.76 & -1.10 & 2.21 & -0.86 & 1.54 & 0.44 \\
\hline Wine 2 & -0.56 & -0.20 & -0.77 & 0.30 & -0.28 & -0.13 & -0.61 & -0.76 \\
\hline Wine 3 & -2.32 & -0.83 & -1.99 & 0.81 & -2.11 & 0.50 & -2.85 & -3.80 \\
\hline Wine 4 & -1.83 & 0.90 & -1.98 & 0.93 & -2.39 & 1.23 & -1.12 & 0.56 \\
\hline Wine 5 & 1.40 & 0.05 & 1.29 & -0.62 & 1.49 & -0.49 & 1.43 & 1.27 \\
\hline Wine 6 & 1.13 & 0.58 & 0.69 & -0.30 & 1.08 & -0.24 & 1.62 & 2.28 \\
\hline
\end{tabular}

sup = supplementary element

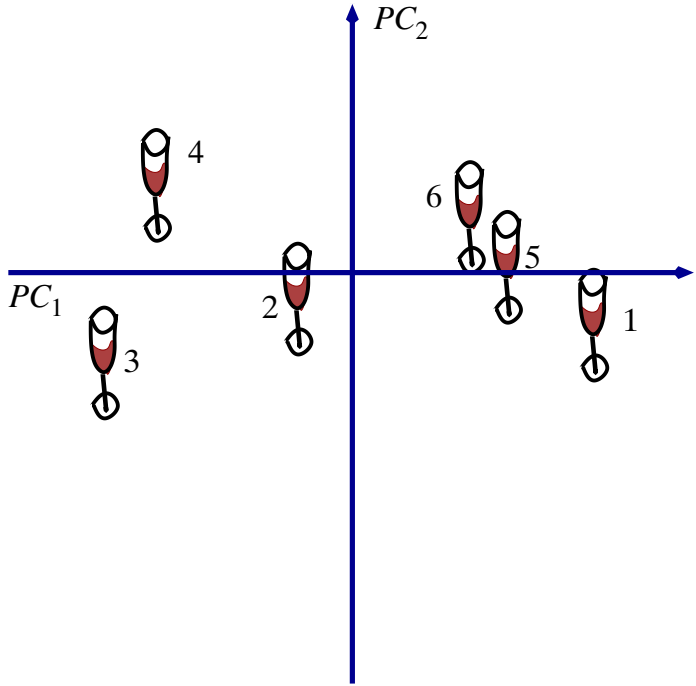

(a)

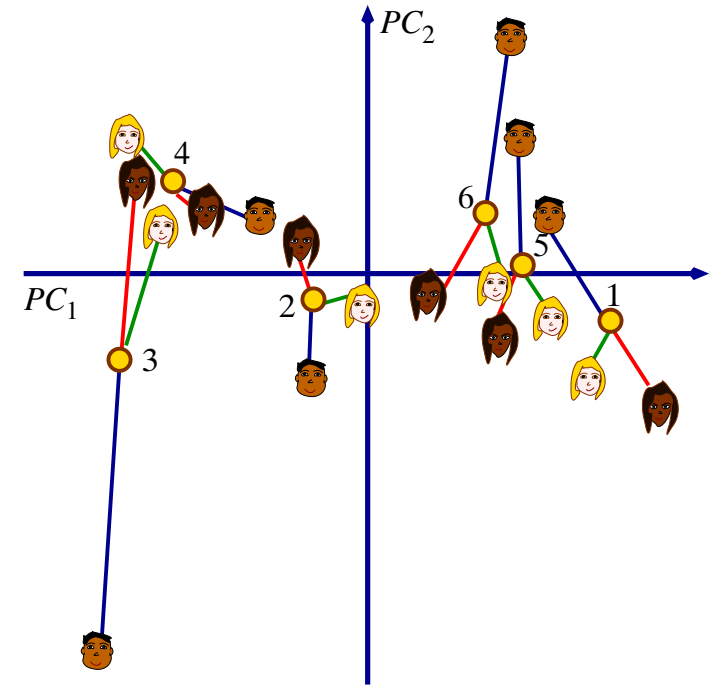

(b)

Figure 11: MFA wine ratings and oak type. (a) Plot of the global analysis of the wines on the first two principal components. (b) Projection of the experts onto the global analysis. Experts are represented by their faces. A line segment links the position of the wine for a given expert to its global position. $\lambda_{1}=2.83, \tau_{1}=84 \% ; \lambda_{2}=2.83$, $\tau_{2}=11 \%$.

The variable loadings show the correlations between the original variables and the global factor scores (Table 19). These loadings are plotted in Figure 12. This figure also represents the loadings (Table 21) between the components of each subtable and the components of the global analysis as the "circle of correlations" specific to each expert. From this we see that Expert 3 differs from the other experts, and is mostly responsible for the second component of the global PCA. 

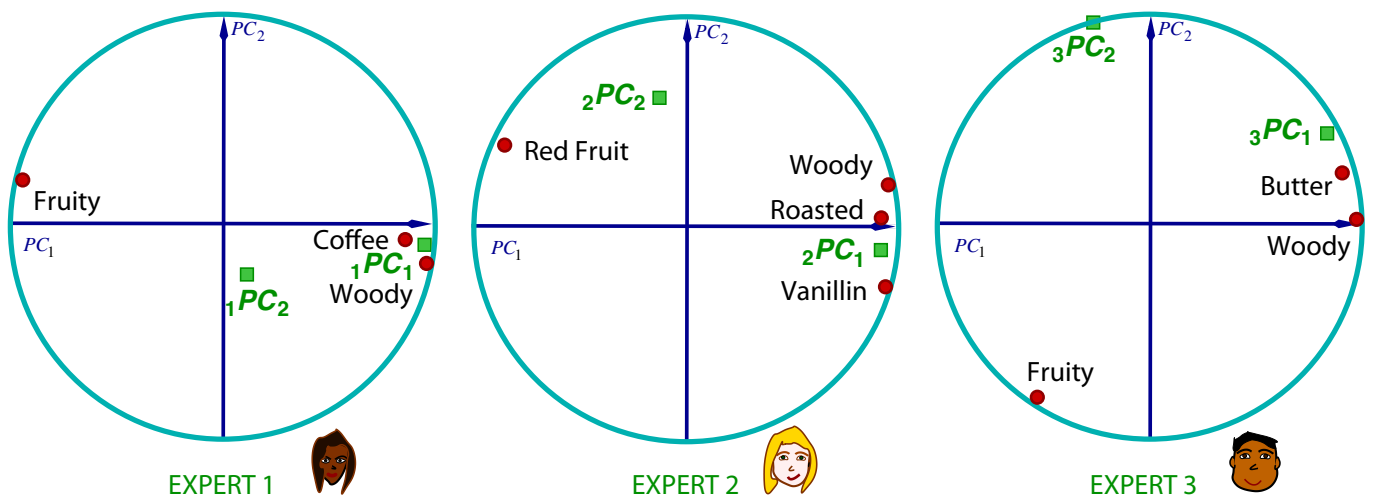

Figure 12: MFA wine ratings and oak type. Circles of correlations for the original variables. Each experts' variables have been separated for ease of interpretation.

Table 21: MFA wine ratings and oak type. Loadings (i.e., correlations) on the principal components of the global analysis of the principal components of the subtable PCA's. Only the first three dimensions are kept.

\begin{tabular}{|c|c|c|c|c|c|c|c|c|}
\hline \multirow[b]{3}{*}{$\mathrm{PC}$} & \multirow[b]{3}{*}{$\lambda$} & \multirow[b]{3}{*}{$\tau(\%)$} & \multicolumn{6}{|c|}{ Loadings with first 2 components from subtable PCA's } \\
\hline & & & \multicolumn{2}{|c|}{ Expert 1} & \multicolumn{2}{|c|}{ Expert 2} & \multicolumn{2}{|c|}{ Expert 3} \\
\hline & & & {$[1] \mathrm{PC}_{1}$} & {$[1] \mathrm{PC}_{2}$} & ${ }_{[2]} \mathrm{PC}_{1}$ & {$[2]{ }^{\mathrm{PC}_{2}}$} & {$[3]^{\mathrm{PC}_{1}}$} & {$[3]^{\mathrm{PC}_{2}}$} \\
\hline 1 & 2.83 & 85 & .98 & .08 & .99 & -.16 & .94 & -.35 \\
\hline 2 & .36 & 11 & -.15 & -.28 & -.13 & -.76 & .35 & .94 \\
\hline 3 & .12 & 3 & -.14 & .84 & .09 & .58 & .05 & -.01 \\
\hline
\end{tabular}

\section{Conclusion}

PCA is very versatile, it is the oldest and remains the most popular technique in multivariate analysis. In addition to the basics presented here, PCA can also be interpreted as a neural network model (see, e.g., Diamantaras and Kung, 1996; Abdi, Valentin, and Edelman, 1999). In addition to correspondence analysis, covered in this paper, generalized PCA can also be shown to incorporate a very large set of multivariate techniques such as canonical variate analysis, linear discriminant analysis (see, e.g., Greenacre, 1984), and barycentric discriminant analysis techniques such as discriminant correspondence analysis (see e.g., Nakache, Lorente, Benzécri, and Chastang, 1977; Saporta and Niang, 2006; Abdi, 2007d; Abdi and Williams, in press-a). 


\section{References}

Abdi, H. (2003a). Factor rotations. In M. Lewis-Beck, A. Bryman, and T. Futing (Eds.), Encyclopedia for research methods for the social sciences. Thousand Oaks, CA: Sage.

Abdi, H. (2003b). Multivariate analysis. In M. Lewis-Beck, A. Bryman, and T. Futing (Eds), Encyclopedia for research methods for the social sciences. Thousand Oaks, CA: Sage.

Abdi, H. (2007a). Singular Value Decomposition (SVD) and Generalized Singular Value Decomposition(GSVD). In N.J. Salkind (Ed), Encyclopedia of measurement and statistics (pp. 907-912). Thousand Oaks: Sage.

Abdi, H. (2007b). Eigen-decomposition: eigenvalues and eigenvectors. In N.J. Salkind (Ed), Encyclopedia of measurement and statistics (pp. 304-308). Thousand Oaks: Sage.

Abdi, H. (2007c). RV cofficient and congruence coefficient. In N.J. Salkind (Ed), Encyclopedia of measurement and statistics (pp. 849-853). Thousand Oaks: Sage.

Abdi, H. (2007d). Discriminant correspondence analysis. In N.J. Salkind (Ed), Encyclopedia of measurement and statistics (pp. 270-275). Thousand Oaks: Sage.

Abdi, H. (in press, 2009). Centroid. Wiley Interdisciplinary Reviews: Computational Statistics, 1.

Abdi, H. (in press, 2010). Partial least square regression, Projection on latent structures Regression, PLS-Regression. Wiley Interdisciplinary Reviews: Computational Statistics, 2.

Abdi, H., and Valentin, D. (2006). Mathématiques pour les sciences cognitives [Mathematics for cognitive sciences]. Grenoble: PUG.

Abdi, H., and Valentin, D. (2007a). Multiple correspondence analysis. In N.J. Salkind (Ed), Encyclopedia of measurement and statistics (pp. 651-657). Thousand Oaks, CA: Sage.

Abdi, H., and Valentin, D. (2007b). Multiple factor analysis (MFA). In N.J. Salkind (Ed), Encyclopedia of measurement and statistics (pp. 657-663). Thousand Oaks, CA: Sage.

Abdi, H., Valentin, D., and Edelman, B. (1999). Neural networks. Thousand Oaks: Sage.

Abdi, H., and Williams, L.J. (in Press, 2010a). Barycentric discriminant analyis (BADIA). In N.J. Salkind (Ed), Encyclopedia of research design. Thousand Oaks: Sage.

Abdi, H., and Williams, L.J. (in Press, 2010b). Correspondence analysis. In N.J. Salkind (Ed), Encyclopedia of research design. Thousand Oaks: Sage.

Abdi, H., and Williams, L.J. (in Press, 2010c). Jackknife. In N.J. Salkind (Ed), Encyclopedia of research design. Thousand Oaks: Sage. 
Baskilevsky, A. (1983). Applied matrix algebra in the statistical sciences. New York: Elsevier.

Benzécri, J.-P. (1973). L'analyse des données, Vols. 1 and 2. Paris: Dunod.

Boyer, C., and Merzbach, U. (1989). A history of mathematics (2nd Edition). New York: Wiley.

Brunet, E. (1989). Faut-il pondérer les données linguistiques. CUMFID, 16, 39-50.

Cattell, R.B. (1966). The scree test for the number of factors. Multivariate Behavioral Research, 1, 245-276.

Cauchy, A.L. (1829). Sur l'équation à l'aide de laquelle on détermine les inégalités séculaires des mouvements des planètes. In Oeuvres Complètes (IIème Série), 9.

Diaconis, P., and Efron, B. (1983). Computer intensive methods in statistics. Scientific American, 248, 116-130.

Diamantaras, K.I., and Kung, S.Y. (1996). Principal component neural networks: Theory and applications. New York: Wiley.

Dray, S. (2008). On the number of principal components: A test of dimensionality based on measurements of similarity between matrices. Computational statistics and Data Analysis, 52, 2228-2237.

Eastment, H.T., and Krzanowski, W.J. (1982). Cross-validatory choice of the number of components from a principal component analysis. Technometrics, 24, 7377 .

Eckart, C., and Young, G. (1936). The approximation of a matrix by another of a lower rank. Psychometrika, 1, 211-218.

Efron, B. (1982). The jackknife, the bootstrap and other resampling plans. Volume 83 of CMBF-NSF Regional Conference Series in Applied Mathematics: SIAM.

Efron, B., and Tibshirani, R.J. (1993). An introduction to the bootstrap. Chapman and Hall: New York.

Escofier, B., and Pagès, J. (1990). Analyses factorielles simples et multiples: objectifs, méthodes, interprétation. Dunod: Paris.

Escofier, B., and Pagès, J. (1994). Multiple factor analysis. Computational Statistics and Data Analysis, 18, 121-140.

Geisser, S. (1974). A predictive approach to the random effect model. Biometrika, 61, 101-107.

Good, I., (1969). Some applications of the singular value decomposition of a matrix. Technometrics, 11, 823-831.

Gower, J. (1971). Statistical methods of comparing different multivariate analyses of the same data. In F. Hodson, D. Kendall, and P. Tautu (Eds.), Mathemematics in the archeological and historical sciences (pp. 138-149). Edinburgh: Edingburh University Press.

Grattan-Guinness, I. (1997). The rainbow of mathematics. New York: Norton.

Greenacre, M.J. (1984). Theory and applications of correspondence analysis. London: Academic Press.

Greenacre, M.J. (2007). Correspondence analysis in practice (2nd Edition). Boca Raton (FL): Chapman \& Hall/CRC. 
Harris, R.J. (2001). A primer of multivariate statistics. Mahwah (NJ): Erlbaum.

Holmes, S. (1989). Using the bootstrap and the $R_{v}$ coefficient in the multivariate context. In E. Diday (Ed.), Data analysis, learning, symbolic and numeric knowledge (pp. 119-132). New York: Nova Science.

Hotelling, H. (1933). Analysis of a complex of statistical variables into principal components. Journal of Educational Psychology, 25, 417-441.

Hwang, H., Tomiuk, M. A., and Takane, Y. (in press). Correspondence analysis, multiple correspondence analysis and recent developments. In R. Millsap and A. Maydeu-Olivares (Eds.). Handbook of quantitative methods in psychology. London: Sage Publications.

Jackson, D.A. (1993). Stopping rules in principal aomponents analysis: A comparison of heuristical and statistical approaches. Ecology, 74, 2204-2214.

Jackson, D.A. (1995). Bootstrapped principal components analysis: A reply to Mehlman et al. Ecology, 76, 644-645.

Jackson, J.E. (1991). A user's guide to principal components. New York: Wiley.

Jordan, C. (1874). Mémoire sur les formes bilinéaires. Journal de Mathématiques Pures et Appliquées, 19, 35-54.

Jolliffe, I.T. (2002). Principal component analysis. New York: Springer.

Kaiser, H.F. (1958). The VARIMAX criterion for analytic rotation in factor analysis. Psychometrika, 23, 187-200.

Kaiser, H.F. (1961). A note on Guttman's lower bound for the number of common factors. British Journal of Mathematical and Statistical Psychology, 14, 1-2.

Kruskal, J.B. (1978). factor analysis and principal component analysis: Bilinear methods. In W.H. Kruskal and J.M. Tannur (Eds.): International Encyclopedia of Statistics. (pp. 307-330). New York: The Free Press.

Lebart, L., and Fénelon, J.P. (1975). Statistique et informatique appliquées. Paris: Dunod.

Lingoes, J., and Schönemann P. (1974). Alternative measures of fit for the Schönemann-Carrol matrix fitting algorithm. Psychometrika, 39, 423-427.

Nakache, J.P., Lorente, P., Benzécri, J.P., and Chastang, J.F. (1977). Aspect pronostics et thérapeutiques de l'infarctus myocardique aigu. Les Cahiers de l'Analyse des Données, 2, 415-534.

Mehlman, D.W., Sheperd, U.L., and Kelt, D.A. (1995). Bootstrapping principal components analysis: A comment. Ecology, 76, 640-643.

Malinowski, E.R. (2002). Factor analysis in chemistry (3rd. Edition). New York: Wiley.

O'Toole, A.J., Abdi, H., Deffenbacher, K.A., and Valentin, D. (1993). A low dimensional representation of faces in the higher dimensions of the space. Journal of the Optical Society of America, Series A, 10, 405-411.

Pearson, K. (1901). On lines and planes of closest fit to systems of points in space. Philosophical Magazine, 6, 559-572.

Peres-Neto, P.R., Jackson, D.A., and Somers, K.M. (2005) How many principal components? stopping rules for determining the number of non-trivial axes re- 
visited. Computational Statistics and Data Analysis, 49, 974-997.

Quenouille, M. (1956). Notes on bias and estimation. Biometrika, 43, 353-360.

Saporta, G, and Niang, N. (2006). Correspondence analysis and classification. In

M. Greenacre and J. Blasius (Eds), Multiple correspondence analysis and related methods. (pp. 371-392). Boca Raton: Chapman \& Hall.

Saporta, G, and Niang, N. (2009). Principal component analysis: application to statistical process control. In G. Govaert (Ed), Data analysis. (pp. 1-23). London: Wiley.

Stewart, G.W. (1993). On the early history of the singular value decomposition. SIAM Review, 35, 551-566.

Stone, M. (1974). Cross-validatory choice and assesment of statistical prediction. Journal of the Royal Statistical Society, Series B, 36, 111-133.

Stone, J.V. (2004). Independent component analysis: A tutorial introduction. Cambridge: MIT Press.

Strang, G. (2003). Introduction to linear algebra. Cambridge, MA: WellesleyCambridge Press.

Takane, Y. (2002). Relationships among various kinds of eigenvalue and singular value decompositions. In Yanai, H., Okada, A., Shigemasu, K., Kano, Y., and Meulman, J. (Eds.), New developments in psychometrics (pp. 45-56). Tokyo: Springer Verlag.

Tennenhaus, M. (1998). La régression PLS. Paris: Technip.

Thurstone, L.L. (1947). Multiple factor analysis. Chicago: University of Chicago Press.

Wold, S. (1995). PLS for Multivariate linear modeling. In H. van de Waterbeemd (Ed.), Chemometric methods in molecular design (pp. 195-217). Weinheim: Wiley-VCH Verlag.

Wold, S. (1978). Cross-validatory estimation of the number of components in factor and principal component analysis. Technometrics, 20, 397-405.

\section{A Eigenvectors, and eigenvalues}

Eigenvectors and eigenvalues are numbers and vectors associated to square matrices. Together they provide the eigen-decomposition of a matrix, which analyzes the structure of this matrix. Even though the eigen-decomposition does not exist for all square matrices, it has a particularly simple expression for matrices such as correlation, covariance, or cross-product matrices. The eigen-decomposition of this type of matrices is important because it is used to find the maximum (or minimum) of functions involving these matrices. Specifically PCA is obtained from the eigen-decomposition of a covariance or a correlation matrix. 


\section{A.1 Notations and definition}

There are several ways to define eigenvectors and eigenvalues, the most common approach defines an eigenvector of the matrix $\mathbf{A}$ as a vector $\mathbf{u}$ that satisfies the following equation:

$$
\mathbf{A u}=\lambda \mathbf{u} .
$$

When rewritten, the equation becomes:

$$
(\mathbf{A}-\lambda \mathbf{I}) \mathbf{u}=\mathbf{0},
$$

where $\lambda$ is a scalar called the eigenvalue associated to the eigenvector.

In a similar manner, we can also say that a vector $\mathbf{u}$ is an eigenvector of a matrix $\mathbf{A}$ if the length of the vector (but not its direction) is changed when it is multiplied by $\mathbf{A}$.

For example, the matrix:

$$
\mathbf{A}=\left[\begin{array}{ll}
2 & 3 \\
2 & 1
\end{array}\right]
$$

has for eigenvectors:

$$
\mathbf{u}_{1}=\left[\begin{array}{l}
3 \\
2
\end{array}\right] \quad \text { with eigenvalue } \quad \lambda_{1}=4
$$

and

$$
\mathbf{u}_{2}=\left[\begin{array}{r}
-1 \\
1
\end{array}\right] \quad \text { with eigenvalue } \quad \lambda_{2}=-1
$$

For most applications we normalize the eigenvectors (i.e., transform them such that their length is equal to one), therefore

$$
\mathbf{u}^{\top} \mathbf{u}=1
$$

Traditionally, we put the set of eigenvectors of $\mathbf{A}$ in a matrix denoted $\mathbf{U}$. Each column of $\mathbf{U}$ is an eigenvector of $\mathbf{A}$. The eigenvalues are stored in a diagonal matrix (denoted $\boldsymbol{\Lambda}$ ), where the diagonal elements gives the eigenvalues (and all the other values are zeros). We can rewrite the Equation 33 as:

$$
\mathrm{AU}=\Lambda \mathrm{U} ;
$$

or also as:

$$
\mathbf{A}=\mathbf{U} \boldsymbol{\Lambda} \mathbf{U}^{-1}
$$


For the previous example we obtain:

$$
\begin{aligned}
\mathbf{A} & =\mathbf{U} \boldsymbol{\Lambda} \mathbf{U}^{-1} \\
& =\left[\begin{array}{lr}
3 & -1 \\
2 & 1
\end{array}\right]\left[\begin{array}{rr}
4 & 0 \\
0 & -1
\end{array}\right]\left[\begin{array}{rr}
2 & 2 \\
-4 & 6
\end{array}\right] \\
& =\left[\begin{array}{ll}
2 & 3 \\
2 & 1
\end{array}\right] .
\end{aligned}
$$

Together, the eigenvectors and the eigenvalues of a matrix constitute the eigendecomposition of this matrix. It is important to note that not all matrices have an eigen-decomposition. This is the case, for example, of the matrix $\left[\begin{array}{ll}0 & 1 \\ 0 & 0\end{array}\right]$. Also some matrices can have imaginary eigenvalues and eigenvectors.

\section{A. 2 Positive (semi-)definite matrices}

A type of matrices used very often in statistics are called positive semi-definite. The eigen-decomposition of these matrices always exists, and has a particularly convenient form. A matrix is said to be positive semi-definite when it can be obtained as the product of a matrix by its transpose. This implies that a positive semi-definite matrix is always symmetric. So, formally, the matrix $\mathbf{A}$ is positive semi-definite if it can be obtained as:

$$
\mathbf{A}=\mathbf{X X}^{\top}
$$

for a certain matrix $\mathbf{X}$ (containing real numbers). In particular, correlation matrices, covariance, and cross-product matrices are all positive semi-definite matrices.

The important properties of a positive semi-definite matrix is that its eigenvalues are always positive or null, and that its eigenvectors are pairwise orthogonal when their eigenvalues are different. The eigenvectors are also composed of real values (these last two properties are a consequence of the symmetry of the matrix, for proofs see, e.g., Strang, 2003; or Abdi and Valentin, 2006). Because eigenvectors corresponding to different eigenvalues are orthogonal, it is possible to store all the eigenvectors in an orthogonal matrix (recall that a matrix is orthogonal when the product of this matrix by its transpose is a diagonal matrix).

This implies the following equality:

$$
\mathbf{U}^{-1}=\mathbf{U}^{\top}
$$


We can, therefore, express the positive semi-definite matrix $\mathbf{A}$ as:

$$
\mathbf{A}=\mathbf{U} \boldsymbol{\Lambda} \mathbf{U}^{\top} \quad \text { with } \quad \mathbf{U}^{\top} \mathbf{U}=\mathbf{I}
$$

where $\mathbf{U}$ is the matrix storing the normalized eigenvectors; if these are not normalized then $\mathbf{U}^{\top} \mathbf{U}$ is a diagonal matrix.

For example, the matrix:

$$
\mathbf{A}=\left[\begin{array}{ll}
3 & 1 \\
1 & 3
\end{array}\right]
$$

can be decomposed as:

$$
\begin{aligned}
\mathbf{A} & =\mathbf{U} \boldsymbol{\Lambda} \mathbf{U}^{\top} \\
& =\left[\begin{array}{ll}
\sqrt{\frac{1}{2}} & \sqrt{\frac{1}{2}} \\
\sqrt{\frac{1}{2}} & -\sqrt{\frac{1}{2}}
\end{array}\right]\left[\begin{array}{ll}
4 & 0 \\
0 & 2
\end{array}\right]\left[\begin{array}{ll}
\sqrt{\frac{1}{2}} & \sqrt{\frac{1}{2}} \\
\sqrt{\frac{1}{2}} & -\sqrt{\frac{1}{2}}
\end{array}\right] \\
& =\left[\begin{array}{ll}
3 & 1 \\
1 & 3
\end{array}\right]
\end{aligned}
$$

with

$$
\left[\begin{array}{cc}
\sqrt{\frac{1}{2}} & \sqrt{\frac{1}{2}} \\
\sqrt{\frac{1}{2}} & -\sqrt{\frac{1}{2}}
\end{array}\right]\left[\begin{array}{cc}
\sqrt{\frac{1}{2}} & \sqrt{\frac{1}{2}} \\
\sqrt{\frac{1}{2}} & -\sqrt{\frac{1}{2}}
\end{array}\right]=\left[\begin{array}{ll}
1 & 0 \\
0 & 1
\end{array}\right] .
$$

\section{A.3 Statistical properties of the eigen-decomposition}

The eigen-decomposition is important because it is involved in problems of optimization. Specifically, in principal component analysis, we want to find row factor scores, obtained as linear combinations of the columns of $\mathbf{X}$ such that these factor scores "explain" as much of the variance of $\mathbf{X}$ as possible and such that the sets of factor scores are pairwise orthogonal. We impose as a constraint that the coefficients of the linear combinations are finite and this constraint is, in general, expressed as imposing to the sum of squares of the coefficients of each linear combination to be equal to unity. This amounts to defining the factor score matrix as

$$
\mathbf{F}=\mathrm{XQ}
$$

(with the matrix $\mathbf{Q}$ being the matrix of coefficients of the "to-be-found" linear combinations) under the constraints that

$$
\mathbf{F}^{\top} \mathbf{F}=\mathbf{Q}^{\top} \mathbf{X}^{\top} \mathrm{XQ}
$$


is a diagonal matrix (i.e., $\mathbf{F}$ is an orthogonal matrix) and that

$$
\mathbf{Q}^{\top} \mathbf{Q}=\mathbf{I}
$$

(i.e., $\mathbf{Q}$ is an orthonormal matrix). The solution of this problem can be obtained with the technique of the Lagrangian multipliers where the constraint from Equation 50 is expressed as the multiplication with a diagonal matrix of Lagrangian multipliers denoted $\boldsymbol{\Lambda}$ in order to give the following expression

$$
\Lambda\left(\mathrm{Q}^{\top} \mathrm{Q}-\mathrm{I}\right)
$$

(see Harris, 2001; and Abdi and Valentin, 2006; for details). This amount to defining the following equation

$$
\mathcal{L}=\operatorname{trace}\left\{\mathbf{F}^{\top} \mathbf{F}-\mathbf{\Lambda}\left(\mathbf{Q}^{\top} \mathbf{Q}-\mathbf{I}\right)\right\}=\operatorname{trace}\left\{\mathbf{Q}^{\top} \mathbf{X}^{\top} \mathbf{X} \mathbf{Q}-\mathbf{\Lambda}\left(\mathbf{Q}^{\top} \mathbf{Q}-\mathbf{I}\right)\right\}
$$

(where the trace \{\} operator gives the sum of the diagonal elements of a square matrix). In order to find the values of $\mathbf{Q}$ which give the maximum values of $\mathcal{L}$, we first compute the derivative of $\mathcal{L}$ relative to $\mathrm{Q}$ :

$$
\frac{\partial \mathcal{L}}{\partial \mathbf{Q}}=2 \mathbf{X}^{\top} \mathbf{X Q}-2 \mathbf{Q} \boldsymbol{\Lambda}
$$

and then set this derivative to zero:

$$
\mathrm{X}^{\top} \mathrm{XQ}-\mathrm{Q} \Lambda=0 \Longleftrightarrow \mathrm{X}^{\top} \mathrm{XQ}=\mathrm{Q} \Lambda
$$

This implies also that

$$
\mathbf{X}^{\top} \mathbf{X}=\mathbf{Q} \Lambda \mathbf{Q}^{\top}
$$

Because $\boldsymbol{\Lambda}$ is diagonal, this is clearly an eigen-decomposition problem, and this indicates that $\Lambda$ is the matrix of eigenvalues of the positive semi-definite matrix $\mathbf{X}^{\top} \mathbf{X}$ ordered from the largest to the smallest and that $\mathbf{Q}$ is the matrix of eigenvectors of $\mathbf{X}^{\top} \mathbf{X}$ associated to $\boldsymbol{\Lambda}$. Finally, we find that the factor matrix has the form

$$
\mathbf{F}=\mathrm{XQ} \text {. }
$$

The variance of the factors scores is equal to the eigenvalues because:

$$
\mathbf{F}^{\top} \mathbf{F}=\mathrm{Q}^{\top} \mathrm{X}^{\top} \mathrm{XQ}=\mathrm{Q}^{\top} \mathrm{Q} \Lambda \mathrm{Q}^{\top} \mathrm{Q}=\boldsymbol{\Lambda}
$$

Taking into account that the sum of the eigenvalues is equal to the trace of $\mathbf{X}^{\top} \mathbf{X}$, this shows that the first factor scores "extract" as much of the variance of the original data as possible, and that the second factor scores extract as much of the variance left unexplained by the first factor, and so on for the remaining factors. Incidently, the diagonal elements of the matrix $\Lambda^{\frac{1}{2}}$ which are the standard deviations of the factor scores are called the singular values of matrix $\mathbf{X}$ (see section on singular value decomposition). 


\section{B Singular Value Decomposition}

The singular value decomposition (SVD) is a generalization of the eigen-decomposition. The SVD decomposes a rectangular matrix into three simple matrices: two orthogonal matrices and one diagonal matrix. If $\mathbf{A}$ is a rectangular matrix, its SVD gives

$$
\mathbf{A}=\mathbf{P} \Delta \mathbf{Q}^{\top}
$$

with

- $\mathbf{P}$ : the (normalized) eigenvectors of the matrix $\mathbf{A} \mathbf{A}^{\top}$ (i.e., $\mathbf{P}^{\top} \mathbf{P}=\mathbf{I}$ ). The columns of $\mathbf{P}$ are called the left singular vectors of $\mathbf{A}$.

- $\mathbf{Q}$ : the (normalized) eigenvectors of the matrix $\mathbf{A}^{\top} \mathbf{A}$ (i.e., $\mathbf{Q}^{\top} \mathbf{Q}=\mathbf{I}$ ). The columns of $\mathbf{Q}$ are called the right singular vectors of $\mathbf{A}$.

$-\Delta$ : the diagonal matrix of the singular values, $\boldsymbol{\Delta}=\boldsymbol{\Lambda}^{\frac{1}{2}}$ with $\boldsymbol{\Lambda}$ being the diagonal matrix of the eigenvalues of matrix $\mathbf{A} \mathbf{A}^{\top}$ and of the matrix $\mathbf{A}^{\top} \mathbf{A}$ (as they are the same).

The singular value decomposition is a straightforward consequence of the eigendecomposition of positive semi-definite matrices (see, e.g., Abdi, 2007a; Greenacre, 1984; Good, 1969; Stewart, 1993).

Note that Equation 58 can also be rewritten as

$$
\mathbf{A}=\mathbf{P} \Delta \mathbf{Q}^{\top}=\sum_{\ell=1}^{L} \delta_{\ell} \mathbf{p}_{\ell} \mathbf{q}_{\ell}^{\top},
$$

with $L$ being the rank of $\mathbf{X}$ and $\delta_{\ell}, \mathbf{p}_{\ell}$, and $\mathbf{q}_{\ell}$ being (respectively) the $\ell$ th singular value, left and right singular vectors of $\mathbf{X}$. This shows that $\mathbf{X}$ can be reconstituted as a sum of $L$ rank one matrices (i.e., the $\delta_{\ell} \mathbf{p}_{\ell} \mathbf{q}_{\ell}^{\top}$ terms). The first of these matrices gives the best reconstitution of $\mathbf{X}$ by a rank one matrix, the sum of the first two matrices gives the best reconstitution of $\mathbf{X}$ with a rank two matrix, and so on, and, in general, the sum of the first $M$ matrices gives the best reconstitution of $\mathbf{X}$ with a matrix of rank $M$.

\section{B.1 Generalized singular value decomposition}

The generalized SVD (GSVD) decomposes a rectangular matrix and takes into account constraints imposed on the rows and the columns of the matrix. The GSVD gives a weighted generalized least square estimate of a given matrix by a lower rank matrix For a given $I \times J$ matrix $\mathbf{A}$, generalizing the singular value decomposition, 
involves using two positive definite square matrices with size $I \times I$ and $J \times J$. These two matrices express constraints imposed on the rows and the columns of A, respectively. Formally, if $\mathbf{M}$ is the $I \times I$ matrix expressing the constraints for the rows of $\mathbf{A}$ and $\mathbf{W}$ the $J \times J$ matrix of the constraints for the columns of $\mathbf{A}$. The matrix $\mathbf{A}$ is now decomposed into:

$$
\mathbf{A}=\widetilde{\mathbf{P}} \widetilde{\Delta} \widetilde{\mathbf{Q}}^{\top} \quad \text { with: } \widetilde{\mathbf{P}}^{\top} \mathbf{M} \widetilde{\mathbf{P}}=\widetilde{\mathbf{Q}}^{\top} \mathbf{W} \widetilde{\mathbf{Q}}=\mathbf{I} .
$$

In other words, the generalized singular vectors are orthogonal under the constraints imposed by $\mathbf{M}$ and $\mathbf{W}$.

This decomposition is obtained as a result of the standard singular value decomposition. We begin by defining the matrix $\widetilde{\mathbf{A}}$ as:

$$
\widetilde{\mathbf{A}}=\mathbf{M}^{\frac{1}{2}} \mathbf{A} \mathbf{W}^{\frac{1}{2}} \Longleftrightarrow \mathbf{A}=\mathbf{M}^{-\frac{1}{2}} \widetilde{\mathbf{A}} \mathbf{W}^{-\frac{1}{2}} \text {. }
$$

We then compute the standard singular value decomposition as $\widetilde{\mathbf{A}}$ as:

$$
\widetilde{\mathbf{A}}=\mathbf{P} \Delta \mathbf{Q}^{\top} \quad \text { with: } \mathbf{P}^{\top} \mathbf{P}=\mathbf{Q}^{\top} \mathbf{Q}=\mathbf{I} .
$$

The matrices of the generalized eigenvectors are obtained as:

$$
\widetilde{\mathbf{P}}=\mathbf{M}^{-\frac{1}{2}} \mathbf{P} \quad \text { and } \quad \widetilde{\mathbf{Q}}=\mathbf{W}^{-\frac{1}{2}} \mathbf{Q} \text {. }
$$

The diagonal matrix of singular values is simply equal to the matrix of singular values of $\widetilde{\mathbf{A}}$ :

$$
\widetilde{\Delta}=\Delta
$$

We verify that:

$$
\mathbf{A}=\widetilde{\mathbf{P}} \widetilde{\Delta} \widetilde{\mathbf{Q}}^{\top}
$$

by substitution:

$$
\begin{aligned}
\mathbf{A} & =\mathbf{M}^{-\frac{1}{2}} \widetilde{\mathbf{A}} \mathbf{W}^{-\frac{1}{2}} \\
& =\mathbf{M}^{-\frac{1}{2}} \mathbf{P} \Delta \mathbf{Q}^{\top} \mathbf{W}^{-\frac{1}{2}} \\
& \left.=\widetilde{\mathbf{P}} \Delta \widetilde{\mathbf{Q}}^{\top} \quad \text { (from Equation } 63\right)
\end{aligned}
$$

To show that Condition 60 holds, suffice it to show that:

$$
\widetilde{\mathbf{P}}^{\top} \mathbf{M} \widetilde{\mathbf{P}}=\mathbf{P}^{\top} \mathbf{M}^{-\frac{1}{2}} \mathbf{M} \mathbf{M}^{-\frac{1}{2}} \mathbf{P}=\mathbf{P}^{\top} \mathbf{P}=\mathbf{I}
$$

and

$$
\widetilde{\mathbf{Q}}^{\top} \mathbf{W} \widetilde{\mathbf{Q}}=\mathbf{Q}^{\top} \mathbf{W}^{-\frac{1}{2}} \mathbf{W} \mathbf{W}^{-\frac{1}{2}} \mathbf{Q}=\mathbf{Q}^{\top} \mathbf{Q}=\mathbf{I}
$$




\section{B.2 Mathematical properties of the singular value decomposition}

It can be shown that the SVD has the important property of giving an optimal approximation of a matrix by another matrix of smaller rank (see, e.g., Good, 1969; Strang, 2003; Abdi and Valentin, 2006). In particular, the SVD gives the best approximation, in a least square sense, of any rectangular matrix by another rectangular matrix of same dimensions, but smaller rank.

Precisely, if $\mathbf{A}$ is an $I \times J$ matrix of rank $L$ (i.e., A contains $L$ singular values that are not zero), we denote by $\mathbf{P}^{[M]}$ (respectively $\mathbf{Q}^{[M]}, \boldsymbol{\Delta}^{[M]}$ ) the matrix made of the first $M$ columns of $\mathbf{P}$ (respectively $\mathbf{Q}, \boldsymbol{\Delta}$ ):

$$
\begin{aligned}
& \mathbf{P}^{[M]}=\left[\mathbf{p}_{1}, \ldots, \mathbf{p}_{m}, \ldots, \mathbf{p}_{M}\right] \\
& \mathbf{Q}^{[M]}=\left[\mathbf{q}_{1}, \ldots, \mathbf{q}_{m}, \ldots, \mathbf{q}_{M}\right] \\
& \boldsymbol{\Delta}^{[M]}=\operatorname{diag}\left\{\delta_{1}, \ldots, \delta_{m}, \ldots, \delta_{M}\right\} .
\end{aligned}
$$

The matrix $\mathbf{A}$ reconstructed from the first $M$ eigenvectors is denoted $\mathbf{A}^{[M]}$. It is obtained as:

$$
\mathbf{A}^{[M]}=\mathbf{P}^{[M]} \boldsymbol{\Delta}^{[M]} \mathbf{Q}^{[M]^{\top}}=\sum_{m}^{M} \delta_{m} \mathbf{p}_{m} \mathbf{q}_{m}^{\top},
$$

(with $\delta_{m}$ being the $m$-th singular value).

The reconstructed matrix $\mathbf{A}^{[M]}$ is said to be optimal (in a least squares sense) for matrices of rank $M$ because it satisfies the following condition:

$$
\left\|\mathbf{A}-\mathbf{A}^{[M]}\right\|^{2}=\operatorname{trace}\left\{\left(\mathbf{A}-\mathbf{A}^{[M]}\right)\left(\mathbf{A}-\mathbf{A}^{[M]}\right)^{\top}\right\}=\min _{\mathbf{X}}\|\mathbf{A}-\mathbf{X}\|^{2}
$$

for the set of matrices $\mathbf{X}$ of rank smaller or equal to $M$ (see, e.g., Eckart and Young, 1936; Good, 1969). The quality of the reconstruction is given by the ratio of the first $M$ eigenvalues (i.e., the squared singular values) to the sum of all the eigenvalues. This quantity is interpreted as the reconstructed proportion or the explained variance, it corresponds to the inverse of the quantity minimized by Equation 71 . The quality of reconstruction can also be interpreted as the squared coefficient of correlation (precisely as the $R_{v}$ coefficient, Abdi, 2007c) between the original matrix and its approximation.

The GSVD minimizes an expression similar to Equation 71, namely

$$
\mathbf{A}^{[M]}=\min _{\mathbf{X}}\left[\operatorname{trace}\left\{\mathbf{M}(\mathbf{A}-\mathbf{X}) \mathbf{W}(\mathbf{A}-\mathbf{X})^{\top}\right\}\right],
$$

for the set of matrices $\mathbf{X}$ of rank smaller or equal to $M$. 